\title{
Interstellar $\mathrm{CN}$ toward $\mathrm{CH}^{+}{ }_{-}$forming regions
}

\author{
R. Gredel ${ }^{1}$, G. Pineau des Forêts ${ }^{2}$, and S. R. Federman ${ }^{3}$ \\ 1 Max-Planck Institut für Astronomie, Königstuhl 17, 69117 Heidelberg, Germany \\ e-mail: gredel@caha.es \\ 2 IAS, Université de Paris-Sud, Bât. 121, 92405 Orsay Cedex, France \\ e-mail: forets@obspm.fr \\ 3 Department of Physics and Astronomy, University of Toledo, Toledo, OH 43606, USA \\ e-mail: sfederm@uoft02.utoledo.edu
}

Received 22 January 2002 / Accepted 8 April 2002

\begin{abstract}
Measurements on interstellar CN absorption are presented for stars in three southern OB associations, NGC 2439, Vela OB1, and Cen OB1. CN is detected in 21 out of 31 stars observed. The doublet ratio for the $R(1)$ and $P(1)$ lines of the $(0,0)$ band of the $\mathrm{B}{ }^{2} \Sigma^{+}-\mathrm{X}^{2} \Sigma^{+}$violet system and a comparison of violet system data with measurements of the $(1,0)$ and $(2,0)$ bands of the $\mathrm{A}{ }^{2} \Pi-\mathrm{X}^{2} \Sigma^{+}$red system are used to derive Doppler parameters and total column densities. Inferred CN column densities vary by more than an order of magnitude for lines of sight with similar $\mathrm{CH}$ column densities. Observations of the $(0,0)$ band of the $\mathrm{CH} \mathrm{B}{ }^{2} \Sigma^{-}-\mathrm{X}^{2} \Pi$ system are used to revise previously published $\mathrm{CH}$ column densities toward the lines of sight studied in CN. Together with earlier results on $\mathrm{CH}, \mathrm{CH}^{+}$, and $\mathrm{C}_{2}$, the $\mathrm{CN}$ data presented here provide a homogeneous set of column densities and radial velocities of diatomic molecules in three individual translucent clouds. We use these data to study $\mathrm{CN}$ production via chemical models. Gas densities are inferred from models based on production via $\mathrm{CH}_{\text {and }} \mathrm{C}_{2}$ in cool gas. Most sightlines in our sample test densities typical for diffuse molecular gas (a few hundred $\mathrm{cm}^{-3}$ ) when the ultraviolet flux permeating the gas is between 1 and 5 times the average interstellar flux. A few lines of sight indicate that $\mathrm{CN}$ is produced under dark cloud conditions because relatively large densities are obtained or because this simple chemical scheme is unable to reproduce the observed CN columns. Low densities are indicated for directions with upper limits on CN. We add an ad hoc component of a number of low-velocity $\left(<10 \mathrm{~km} \mathrm{~s}^{-1}\right)$ criss-crossing MHD shocks to explain observed column densities of interstellar $\mathrm{CH}^{+}$. These shocks also produce about 10 to $30 \%$ of the total $\mathrm{CH}$ column along the line of sight.
\end{abstract}

Key words. ISM: general - ISM: abundances - ISM: clouds - ISM: molecules

\section{Introduction}

In diffuse molecular gas, $\mathrm{CN}$ is a probe of relatively dense regions (e.g., Joseph et al. 1986). A survey of 15 lines of sight with known molecular hydrogen abundances was used to identify the basic reactions which are responsible for the formation of $\mathrm{CN}$ in diffuse clouds (Federman et al. 1984; Federman \& Lambert 1988). For total molecular hydrogen columns of $N\left(\mathrm{H}_{2}\right) \leq 10^{21} \mathrm{~cm}^{-2}$, the column density of $\mathrm{CN}$ is found to correlate with that of $\mathrm{H}_{2}$, with $\log N(\mathrm{CN}) \approx 3 \log N\left(\mathrm{H}_{2}\right)$ (Federman et al. 1984). Toward more reddened lines of sight, the correlation seems to break down. Two studies presented by van Dishoeck \& Black (1989) and Gredel et al. (1991) of

Send offprint requests to: R. Gredel, e-mail: gredel@mpia.de some 27 lines of sight with visual extinctions up to 5 mag showed that the CN column densities may vary by more than an order of magnitude for lines of sight where the $\mathrm{CH}$ column densities are similar. From a comparison of their model calculations with the observations, van Dishoeck \& Black (1989) suggested that the large differences in the $\mathrm{CN} / \mathrm{CH}$ column densities may arise from variations in the intensity of the UV radiation field at $\lambda<1000 \AA$. More recently, Federman et al. (1994) pointed out that the chemistry of $\mathrm{CN}$, and that of $\mathrm{C}_{2}$, is affected by chemical transitions, which take place at grain optical depths $\tau_{\mathrm{UV}}>3$ where neutral-neutral reactions of $\mathrm{N}$ with $\mathrm{CH}$ and $\mathrm{C}_{2}$ dominate $\mathrm{CN}$ production channels, and at $\tau_{\mathrm{UV}} \geq 4.5$ where photodissociation of $\mathrm{CN}$ ceases to be important. The rate equations for $\mathrm{C}_{2}$ and $\mathrm{CN}$ developed by Federman et al. (1994) were used to extract physical conditions in 
photodissociation regions toward stars illuminating reflection nebulae (Federman et al. 1997a; Knauth et al. 2001) and to study density variations over subparsec scales in diffuse molecular gas (Pan et al. 2001). Limits on $\mathrm{CN}$ abundances also place constraints on the amount of $\mathrm{CH}$ produced during $\mathrm{CH}^{+}$synthesis (Federman et al. 1997b; Zsargó 2000). This highlights the different regions probed by $\mathrm{CN}$ and $\mathrm{CH}^{+}$absorption. A goal of the present study is to examine this correspondence for gas toward three southern associations.

A very different aspect of interstellar $\mathrm{CN}$ is its use to determine the cosmic microwave background radiation temperature, $T_{\mathrm{CMB}}$. Very successfully, high signal to noise observations of optical absorption lines have been used to derive a value of $T_{\mathrm{CMB}}=2.73 \mathrm{~K}$ (e.g., Roth \& Meyer 1995, and references therein), which agrees remarkably well with the value of $T_{\mathrm{CMB}}=2.728 \mathrm{~K}$ determined by COBE toward the Galactic North Pole (Fixsen et al. 1997). Collisional impact excitation by electrons may, however, increase the $\mathrm{CN}$ excitation temperature above $T_{\mathrm{CMB}}$ (Black \& van Dishoeck 1991) in clouds where the fractional ionisation is high. The CN rotational excitation temperature which is inferred from the measurements may also be used to judge whether the inferred $\mathrm{CN}$ column densities are reliable or not.

The focus of the present work is to complement previously reported observations of $\mathrm{CH}^{+}, \mathrm{CH}$, and $\mathrm{C}_{2}$ toward the three southern OB associations NGC 2439, Vela OB1, and Cen OB1 (Gredel 1997, 1999). The study of molecular abundances toward members of a given association gives some confidence that variations in the column densities arise from variations in the physical and chemical conditions in spatially coherent structures or clouds. In particular, the variation in the molecular abundances with visual extinction of the background stars may be used to study the dependence of the molecular material on optical depth of a cloud. With the addition of CN presented below, we provide a homogeneous set of column densities and radial velocities of diatomic molecules in three translucent clouds. Our observations focused on the CN absorption lines which arise from the $(0,0)$ band of the $\mathrm{B}^{2} \Sigma^{+}-\mathrm{X}^{2} \Sigma^{+}$ violet system. Additional data on the $(1,0)$ and $(2,0)$ bands of the $\mathrm{CN} \mathrm{A}{ }^{2} \Pi-\mathrm{X}^{2} \Sigma^{+}$red system are used to obtain saturation corrections. The observations are discussed in Sect. 2. The methods used to estimate the CN Doppler parameters, $b(\mathrm{CN})$, and the saturation corrections are described in Sect. 3.1. Our spectra of the CN violet system cover absorption lines which arise from the $(0,0)$ band of the $\mathrm{CH} \mathrm{B}{ }^{2} \Sigma^{-}-\mathrm{X}^{2} \Pi$ system as well. The new $\mathrm{CH}$ data are used to verify the previously inferred $\mathrm{CH}$ column densities (Gredel 1997, hereafter Paper I) and to check whether the saturation corrections which were applied to derive column densities are reasonable or not. The new $\mathrm{CH}$ data are presented in Sects. 2.3 and 3.2. A comparison of the radial velocities of $\mathrm{CN}, \mathrm{CH}^{+}, \mathrm{CH}$, and $\mathrm{C}_{2}$ is given in Sect. 4.1. Derived CN column densities are compared with general predictions from theoretical models and are used to infer gas densities (Sect. 4.2). We aim to explain the observed
$\mathrm{CH}^{+}$column densities by introducing an ad hoc component of some 10-50 criss-crossing MHD shocks per line of sight (Sect. 4.3).

\section{Observations and data reduction}

\subsection{The $(0,0)$ band of the $B^{2} \Sigma^{+}-X^{2} \Sigma^{+} C N$ violet system}

Absorption from the $R(0), R(1)$, and $P(1)$ lines of the $(0,0)$ band of the $\mathrm{CN} \mathrm{B}{ }^{2} \Sigma^{+}-\mathrm{X}{ }^{2} \Sigma^{+}$violet system was sought toward early type stars in the NGC 2439, Vela OB1, and Cen OB1 associations, using the $1.4 \mathrm{~m}$ Coudé Auxiliary Telescope (CAT) on La Silla. The observations were obtained during the nights of April 6-11, 1997 under good atmospheric conditions. The Coudé Echelle Spectrograph (CES) was used at a spectral resolving power of $R=\lambda / \Delta \lambda=60000$. Total exposure times range from $0.5-2$ hours and were set to reach a signalto-noise ratio in the stellar continuum of $S / N \geq 100$ in general. The observations were reduced following standard procedures - see Gredel et al. 1991 for details. The $R(0), R(1)$, and $P(1)$ lines are unresolved blends of two or three spin-rotation components, $\left[R_{1}(0)+{ }^{R} Q_{12}(0)\right]$, $\left[R_{1}(1)+R_{2}(1)+{ }^{R} Q_{12}(1)\right]$, and $\left[P_{1}(1)+{ }^{P} Q_{12}(1)\right]$, with effective rest wavelengths of $3874.602 \AA, 3873.994 \AA$, and $3875.759 \AA$, respectively (Prasad et al. 1992). In the limit of unsaturated lines, the equivalent widths $W_{\lambda}$ are converted into column density $N_{\infty}$ using $N_{\infty}=1.13 \times$ $10^{20} W_{\lambda} / \lambda^{2} f_{J^{\prime} J^{\prime \prime}}$, where $N_{\infty}$ is in units of $\mathrm{cm}^{-2}$ and $W_{\lambda}$ and $\lambda$ are both in $\AA$. Line oscillator strengths $f_{J^{\prime} J^{\prime \prime}}$ are factorised into $f_{J^{\prime} J^{\prime \prime}}=p_{J^{\prime} J^{\prime \prime}} f_{00}$, with values of $p_{J^{\prime} J^{\prime \prime}}$ of $1.0,0.667$, and 0.333 , for the $R(0), R(1)$, and $P(1)$ line blends, respectively (Federman et al. 1984). We adopt the theoretical band oscillator strengths of $f_{00}=0.0342$ of Bauschlicher et al. (1988).

The final spectra for stars in NGC 2439, Vela OB1, and Cen OB1 are shown in Figs. 1-3, respectively. All spectra are normalised to a continuum level of 1 and are in a heliocentric reference frame. $\mathrm{CN}$ absorption lines are detected in all observed stars in NGC 2439, in 7 out of 10 stars in the Vela OB1 Association, and in 8 out of 10 stars in Cen OB1. Note that HD 78344 is considered a probable member of Vela OB1 (Reed 2000). Toward HD 110639, HD 113422, and HD 114213, the CN absorption occurs in 2 well resolved velocity components. Toward HD 61827, the $\mathrm{CN}$ absorption lines are partially resolved into two velocity components. Additional spectra not shown here were taken of HD 54662 in CMa OB1, and of HD 111904, HD 111973, HD 111990, and CPD-59 4551 in NGC 4755; $\mathrm{CN}$ is not detected toward any of these stars and upper limits are $N(\mathrm{CN})<5 \times 10^{11} \mathrm{~cm}^{-2}$.

The measurements are summarised in Tables 1 and 2, with the stellar ID, the line designation, the heliocentric wavelength $\lambda_{\text {hel }}$ in $\AA$, the corresponding heliocentric velocity $V_{\text {hel }}$ in $\mathrm{km} \mathrm{s}^{-1}$, the measured equivalent width $W_{\lambda}$ in $\mathrm{m} \AA$, and the corresponding column density $N_{\infty}\left(N^{\prime \prime}\right)$ inferred in the limit of unsaturated lines in Cols. 1-6, 
NGC 2439

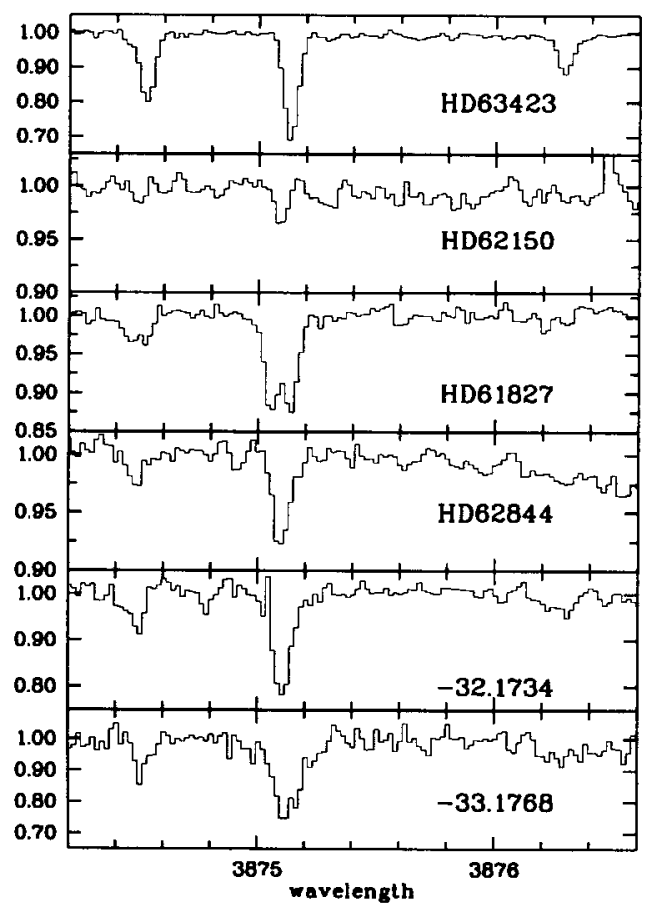

Fig. 1. $R(0), R(1)$, and $P(1)$ absorption lines of the $(0,0)$ band of the $\mathrm{CN} \mathrm{B}{ }^{2} \Sigma^{+}-\mathrm{X}^{2} \Sigma^{+}$violet system toward various stars in the NGC 2439 association.

respectively. Measurement uncertainties in the equivalent widths are dominated by the uncertainties in the placement of the local stellar continuum rather than by the noise in the stellar continuum. The uncertainties in $W_{\lambda}$ and the resulting uncertainties in the inferred column densities are given in parentheses in Cols. 5 and 6 , respectively. The heliocentric velocities $\overline{V_{\text {hel }}}$ listed in Col. 7 are averages, with the oscillator strengths of the corresponding absorption lines used as the weighting factor.

\subsection{The $(1,0)$ and $(2,0)$ bands of the $C N$ $A^{2} \Pi-X^{2} \Sigma^{+}$red system}

Interstellar absorption lines in the $(1,0)$ and $(2,0)$ bands of the $\mathrm{A}^{2} \Pi-\mathrm{X}{ }^{2} \Sigma^{+} \mathrm{CN}$ red system are detected in the echelle spectra of Gredel (1999). Some of the absorption lines of the $\mathrm{CN}$ red system are unresolved blends of spin-rotation components. Relevant line blends studied here are $\left[R_{2}(1)+{ }^{R} Q_{21}(1)\right],\left[Q_{2}(1)+{ }^{Q} P_{21}(1)\right]$, and $\left[{ }^{Q} R_{12}(1)+Q_{1}(1)\right]$, with effective wavelengths near $9142.838 \AA, 9147.203 \AA$, and $9190.113 \AA$, respectively, in the $(1,0)$ band, and $7873.987 \AA, 7877.191 \AA$, and $7908.954 \AA$, respectively, in the $(2,0)$ band. The effective wavelengths are weighted averages obtained from the wavelengths given by van Dishoeck \& Black (1989) for the various spin-rotation components, with the corresponding oscillator strengths used as weight.

The equivalent width of a spin-rotation line blend with contributions from the $\left(N^{\prime \prime}=1, J^{\prime \prime}=\frac{1}{2}\right)$
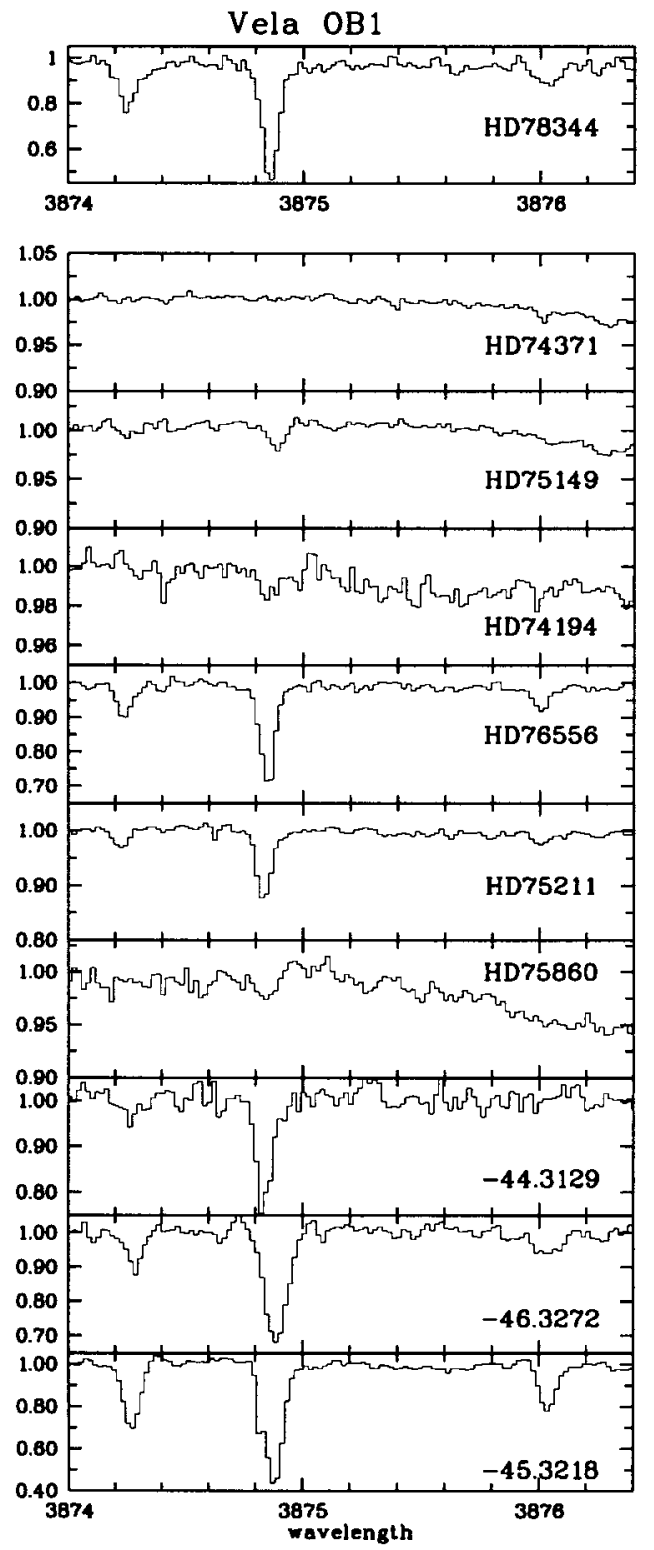

Fig. 2. Same as Fig. 1 for stars in Vela OB1.

and $\left(N^{\prime \prime}=1, J^{\prime \prime}=\frac{3}{2}\right)$ levels is given by $W_{\lambda}=$ $\frac{\pi e^{2}}{m c^{2}} \lambda^{2}\left\{N\left(1, \frac{1}{2}\right) / f_{J^{\prime}, J^{\prime \prime}=\frac{1}{2}}+N\left(1, \frac{3}{2}\right) / f_{J^{\prime}, J^{\prime \prime}=\frac{3}{2}}\right\}$. We assume that the spin-rotational levels $\left(N^{\prime \prime}, J^{\prime \prime}\right)$ with the same rotational quantum number $N^{\prime \prime}$ are populated according to their statistical weights. Total column densities $N(1)=N\left(1, \frac{1}{2}\right)+N\left(1, \frac{3}{2}\right)$ are then given by $N(1)=$ $3 \times N\left(1, \frac{1}{2}\right)$ or $N(1)=1.5 \times N\left(1, \frac{3}{2}\right)$. We obtain $W_{\lambda}=$ $\frac{\pi e^{2}}{m c^{2}} \lambda^{2} N(1) f\left(v^{\prime} v^{\prime \prime}\right) f_{\text {eff }}$, with an effective rotational oscillator strength of $f_{\text {eff }}=\left(f_{J^{\prime} J^{\prime \prime}=\frac{1}{2}} / 3.0+f_{J^{\prime} J^{\prime \prime}=\frac{3}{2}} / 1.5\right)$. As above, we factorise $f_{\text {eff }}=p_{\text {eff }} f\left(v^{\prime} v^{\prime \prime}\right), p_{\text {eff }}=p_{J^{\prime} J^{\prime \prime}=\frac{1}{2}} / 3.0+$ $p_{J^{\prime} J^{\prime \prime}=\frac{3}{2}} / 1.5$, and derive values of $p_{\text {eff }}=0.228,0.167$, and 0.305 , respectively, for the $\left[R_{2}(1)+{ }^{R} Q_{21}(1)\right],\left[Q_{2}(1)+\right.$ $\left.{ }^{Q} P_{21}(1)\right]$, and $\left[{ }^{Q} R_{12}(1)+Q_{1}(1)\right]$ blends. Absorption lines from $N^{\prime \prime}=0$ arise from one spin-rotation component only $\left(J^{\prime \prime}=\frac{1}{2}\right)$. For the $R_{1}(0),{ }^{R} Q_{21}(0)$, and ${ }^{S} R_{21}(0)$ lines, $p_{\text {eff }}=p_{J^{\prime} J^{\prime \prime}=\frac{1}{2}}=0.528,0.334$, and 0.138 , respectively. Column densities are inferred through the use of the 


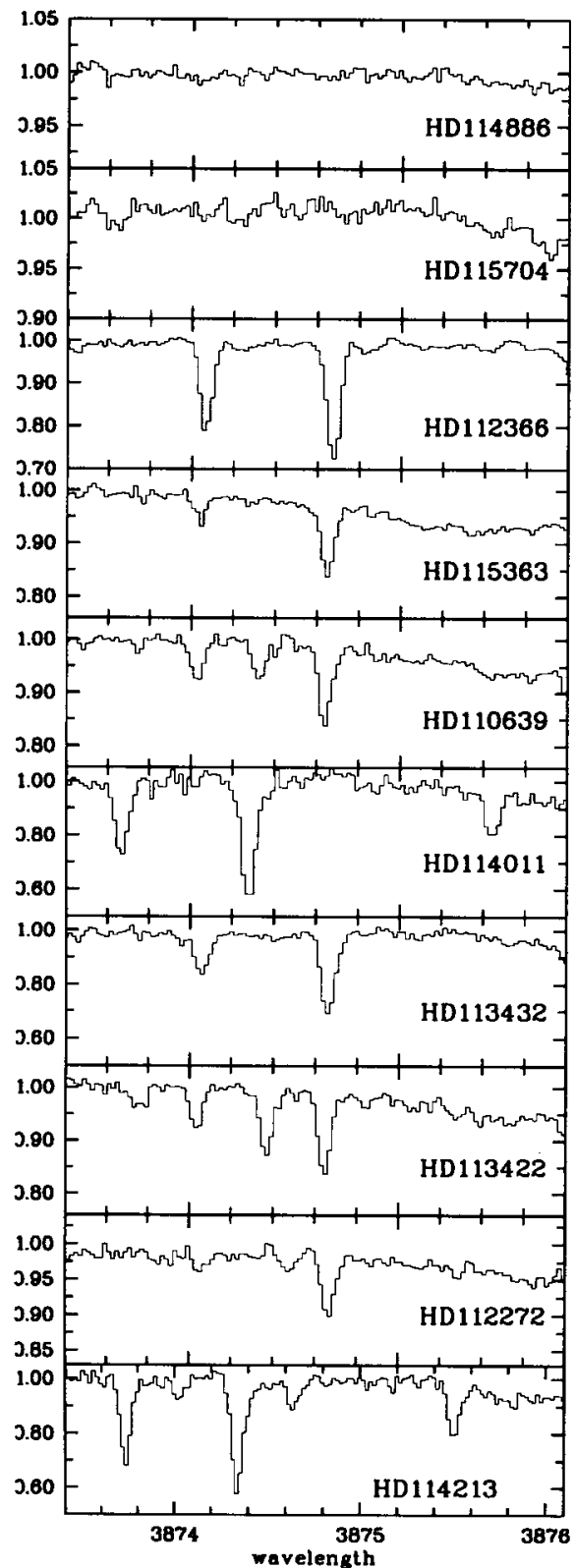

Fig. 3. Same as Fig. 1 for stars in Cen OB1.

experimental band oscillator strengths of $f_{10}=1.5 \times 10^{-3}$ and $f_{20}=7.6 \times 10^{-4}$ of Davis et al. (1986).

The spectra covering the $(2,0)$ and $(1,0)$ bands of the $\mathrm{CN}$ red system are displayed in Figs. 4 and 5, respectively. The spectra are dominated by strong telluric absorption features. Filled symbols identify detected CN lines, and open symbols mark features used to infer upper limits in the CN column densities. Toward CPD-33 1768 , HD 113432, HD 114011, and HD 114213, CN absorption lines are detected in both bands of the red system. A few of the other stars show marginal detections with equivalent widths of $2-3$ standard deviations, mainly in the $R_{1}(0)$ line of both bands. Table 3 summarises the measurements in the $\mathrm{CN}$ red system, with the star, the band and the line designation, the corresponding spin and rotational quantum numbers $J^{\prime \prime}$ and $N^{\prime \prime}$, the heliocen-
Table 1. Data on the CN violet system toward NGC 2439 and Vela $\mathrm{OB}^{a}$

\begin{tabular}{|c|c|c|c|c|c|c|}
\hline $\begin{array}{l}\text { star } \\
\mathrm{HD} / \mathrm{CPD}\end{array}$ & line & $\lambda_{\text {hel }}$ & $V_{\text {hel }}$ & $W_{\lambda}$ & $N_{\infty}\left(N^{\prime \prime}\right)$ & $\overline{V_{\text {hel }}}$ \\
\hline \multicolumn{7}{|l|}{ NGC2439 } \\
\hline \multirow[t]{3}{*}{63423} & $R(1)$ & 3874.526 & 41.1 & $15.0(0.5)$ & $4.9(0.2)$ & \\
\hline & $R(0)$ & 3875.134 & 41.1 & $22.0(0.5)$ & $4.8(0.1)$ & \\
\hline & $P(1)$ & 3876.289 & 40.9 & $8.0(0.5)$ & $5.3(0.3)$ & 41.1 \\
\hline 62150 & $R(0)$ & 3875.092 & 37.8 & $1.5(0.5)$ & $0.3(0.1)$ & 37.8 \\
\hline \multirow[t]{6}{*}{61827} & $R(1)$ & 3874.459 & 35.9 & $2.5(1.0)$ & $0.8(0.3)$ & \\
\hline & $R(0)$ & 3875.059 & 35.3 & $10.0(1.0)$ & $.2)$ & \\
\hline & $P(1)$ & 3876.212 & 35.0 & $1.5(1.0)$ & $1.0(0.7)$ & 35.4 \\
\hline & $R(1)$ & 3874.526 & 41.1 & $2.0(1.0)$ & $0.7(0.3)$ & \\
\hline & $R(0)$ & 3875.145 & 41.9 & $10.0(1.0)$ & & \\
\hline & $P(1)$ & 3876.285 & 40.6 & $.0)$ & .7) & 41.4 \\
\hline \multirow[t]{2}{*}{62844} & $R(1)$ & 3874.482 & 37.7 & $3.0(1.0)$ & $1.0(0.3)$ & \\
\hline & $R(0)$ & 3875.100 & 38.4 & $8.0(1.0)$ & $1.8(0.2)$ & 38.1 \\
\hline \multirow[t]{3}{*}{$-32^{\circ} 1734$} & $R(1)$ & 3874.486 & 38.0 & $6.0(1.5)$ & $2.0(0.5)$ & \\
\hline & $R(0)$ & 3875.108 & 39.1 & $20.5(1.5)$ & & \\
\hline & $P(1)$ & 3876.267 & 39.2 & $6.0(3.0)$ & $4.0(2.0)$ & 38.7 \\
\hline \multirow[t]{3}{*}{$-33^{\circ} 1768$} & $R(1)$ & 3874.518 & 40.5 & $7.5(3.0)$ & $2.5(1.0)$ & \\
\hline & $R(0)$ & 3875.129 & 40.7 & $30.0(3.0)$ & $6.6(0.7)$ & \\
\hline & $P(1)$ & 3876.286 & 40.7 & $5.0(3.0)$ & $3.3(2.0)$ & 40.6 \\
\hline \multicolumn{7}{|l|}{ Vela OB1 } \\
\hline \multirow[t]{3}{*}{78344} & $R(1)$ & 3874.256 & 20.2 & $20.0(3.0)$ & $6.6(1.0)$ & \\
\hline & $R(0)$ & 3874.861 & 20.0 & $46.0(3.0)$ & 10.1 & \\
\hline & $P(1)$ & 3876.030 & 20.9 & $11.0(3.0)$ & $7.3(2.0)$ & 20.2 \\
\hline 75149 & $R(0)$ & 3874.892 & 22.4 & $2.0(0.5)$ & 0.4( & 22.4 \\
\hline \multirow[t]{3}{*}{76556} & $R(1)$ & 3874.240 & 19.0 & $7.5(1.0)$ & $2.5(0.3)$ & \\
\hline & $R(0)$ & 3874.849 & 19.0 & $23.0(1.0)$ & $5.1(0.2)$ & \\
\hline & $P(1)$ & 3876.005 & 19.0 & $6.0(1.0)$ & $4.0(0.7)$ & 19.0 \\
\hline \multirow[t]{3}{*}{75211} & $R(1)$ & 3874.225 & 17.9 & $2.0(1.0)$ & $0.7(0.3)$ & \\
\hline & $R(0)$ & 3874.834 & 17.9 & $12.0(1.0)$ & $2.6(0.2)$ & \\
\hline & $P(1)$ & 3876.010 & 19.4 & $\leq 1.5$ & $\leq 1.0$ & 18.1 \\
\hline$-44^{\circ} 3129$ & $R(0)$ & 3874.836 & 18.0 & $18.0(1.0)$ & $4.0(0.2)$ & 18.0 \\
\hline \multirow[t]{3}{*}{$-46^{\circ} 3272$} & $R(1)$ & 3874.283 & 22.3 & $10.5(2.0)$ & $3.5(0.7)$ & \\
\hline & $R(0)$ & 3874.888 & 22.1 & $39.0(2.0)$ & $8.6(0.4)$ & \\
\hline & $P(1)$ & 3876.032 & 21.1 & $7.0(2.0)$ & $4.6(1.3)$ & 22.0 \\
\hline \multirow[t]{3}{*}{$-45^{\circ} 3218$} & $R(1)$ & 3874.273 & 21.6 & $25.0(3.0)$ & $8.3(1.0)$ & \\
\hline & $R(0)$ & 3874.884 & 21.8 & $49.0(3.0)$ & 10.8( & \\
\hline & $P(1)$ & 3876.039 & 21.6 & $15.5(3.0)$ & $10.2(2.0)$ & 21.7 \\
\hline
\end{tabular}

${ }^{a} \lambda_{\text {hel }}$ in $\AA, V_{\text {hel }}$ in $\mathrm{km} \mathrm{s}^{-1}, W_{\lambda}$ in $\mathrm{m} \AA, N$ in $10^{12} \mathrm{~cm}^{-2}$.

tric wavelength in $\AA$, the corresponding heliocentric velocity in $\mathrm{km} \mathrm{s}^{-1}$, the measured equivalent width $W_{\lambda}$ in $\mathrm{m} \AA$, and the inferred column density $N\left(N^{\prime \prime}, J^{\prime \prime}\right)$, in Cols. $1-8$, respectively. Note that our analysis yields total column densities $N(1)$ for line blends, but column densities in individual spin-rotation levels for single lines such as the $R_{1}(1)$ line. Total column densities in the $N^{\prime \prime}=0$ and $N^{\prime \prime}=1$ rotational levels are given in Cols. 9 and 10, respectively, and are averages of values obtained in the $(1,0)$ and $(2,0)$ bands when available, weighted with the oscillator strengths. The corresponding rotational excitation temperatures $T_{\text {red }}$ are given in Col. 11 of Table 3 . 
Table 2. Data on the $\mathrm{CN}$ violet system toward Cen $\mathrm{OB}^{a}$.

\begin{tabular}{|c|c|c|c|c|c|c|}
\hline $\begin{array}{l}\text { star } \\
\text { HD }\end{array}$ & line & $\lambda_{\text {hel }}$ & $V_{\text {hel }}$ & $W_{\lambda}$ & $N_{\infty}\left(N^{\prime \prime}\right)$ & $\overline{V_{\text {hel }}}$ \\
\hline \multirow[t]{3}{*}{112366} & $R(1)$ & 3874.064 & 5.4 & $15.5(2.0)$ & $5.1(0.7)$ & \\
\hline & $R(0)$ & 3874.671 & 5.3 & $20.0(2.0)$ & $4.4(0.4)$ & \\
\hline & $P(1)$ & 3875.833 & 5.7 & $11.0(2.0)$ & $7.3(1.3)$ & 5.4 \\
\hline \multirow[t]{2}{*}{115363} & $R(1)$ & 3874.044 & 3.9 & $2.0(0.5)$ & $0.7(0.2)$ & \\
\hline & $R(0)$ & 3874.650 & 3.7 & $9.5(0.5)$ & $2.1(0.1)$ & 3.8 \\
\hline \multirow[t]{5}{*}{110639} & $R(1)$ & 3874.031 & 2.9 & $6.0(0.5)$ & $2.0(0.2)$ & \\
\hline & $R(0)$ & 3874.640 & 2.9 & $11.0(0.5)$ & $2.4(0.1)$ & \\
\hline & $P(1)$ & 3875.796 & 2.9 & $2.5(0.5)$ & $1.7(0.3)$ & 2.9 \\
\hline & $R(1)$ & 3873.746 & -19.2 & $1.5(0.5)$ & $0.5(0.2)$ & \\
\hline & $R(0)$ & 3874.324 & -21.5 & $4.5(0.5)$ & $1.0(0.1)$ & -20.6 \\
\hline \multirow[t]{3}{*}{114011} & $R(1)$ & 3873.669 & -25.1 & $22.0(1.0)$ & $7.3(0.3)$ & \\
\hline & $R(0)$ & 3874.280 & -24.9 & $41.5(1.0)$ & $9.1(0.2)$ & \\
\hline & $P(1)$ & 3875.444 & -24.3 & $13.5(1.0)$ & $8.9(0.7)$ & -24.9 \\
\hline \multirow[t]{3}{*}{113432} & $R(1)$ & 3874.057 & 4.9 & $12.0(1.0)$ & $4.0(0.3)$ & \\
\hline & $R(0)$ & 3874.662 & 4.6 & $24.5(1.0)$ & $5.4(0.2)$ & \\
\hline & $P(1)$ & 3875.819 & 4.6 & $5.5(1.0)$ & $3.6(0.7)$ & 4.7 \\
\hline \multirow[t]{5}{*}{113422} & $R(1)$ & 3873.751 & -18.8 & $3.5(1.0)$ & $1.2(0.3)$ & \\
\hline & $R(0)$ & 3874.368 & -18.1 & $7.5(1.0)$ & $1.7(0.2)$ & -18.5 \\
\hline & $R(1)$ & 3874.033 & 3.0 & $5.0(1.0)$ & $1.7(0.3)$ & \\
\hline & $R(0)$ & 3874.647 & 3.4 & $10.5(1.0)$ & $2.3(0.2)$ & \\
\hline & $P(1)$ & 3875.793 & 2.6 & $2.0(1.0)$ & $1.3(0.7)$ & 3.2 \\
\hline \multirow[t]{2}{*}{112272} & $R(1)$ & 3874.054 & 4.6 & $1.5(0.5)$ & $0.5(0.2)$ & \\
\hline & $R(0)$ & 3874.673 & 5.5 & $5.5(0.5)$ & $1.2(0.1)$ & 5.0 \\
\hline \multirow[t]{6}{*}{114213} & $R(1)$ & 3873.734 & -20.1 & $22.0(1.0)$ & $7.3(0.3)$ & \\
\hline & $R(0)$ & 3874.343 & -20.0 & $32.0(1.0)$ & $7.0(0.2)$ & \\
\hline & $P(1)$ & 3875.506 & -19.5 & $14.5(1.0)$ & $9.6(0.7)$ & -20.0 \\
\hline & $R(1)$ & 3874.024 & 2.3 & $1.5(1.0)$ & $0.5(0.3)$ & \\
\hline & $R(0)$ & 3874.642 & 3.1 & $8.0(1.0)$ & $1.8(0.2)$ & \\
\hline & $P(1)$ & 3875.827 & 5.3 & $2.0(1.0)$ & $1.3(0.7)$ & 3.2 \\
\hline
\end{tabular}

${ }^{a} \lambda_{\text {hel }}$ in $\AA, V_{\text {hel }}$ in $\mathrm{km} \mathrm{s}^{-1}, \mathrm{~W}_{\lambda}$ in $\mathrm{m} \AA, \mathrm{N}$ in $10^{12} \mathrm{~cm}^{-2}$.

\subsection{The $(0,0)$ band of the $C H B^{2} \Sigma^{-}-X^{2} \Pi$ system}

The spectra taken with the CAT cover the $R_{2}(1)$ $(3878.774 \AA),\left[Q_{2}(1)+{ }^{Q} R_{12}(1)\right](3886.409 \AA)$, and the ${ }^{P} Q_{12}(1) \quad(3890.217 \AA)$ lines of the $(0,0)$ band of the B ${ }^{2} \Sigma^{-}-\mathrm{X}{ }^{2} \Pi$ system of $\mathrm{CH}$. These additional data are most useful as supplements to previously reported $\mathrm{CH}$ column densities and radial velocities (Gredel 1997). We use the molecular parameters employed by Gredel et al. (1993) to convert measured equivalent widths and wavelengths into column densities and radial velocities. The measurements are summarised in Tables 4 and 5, with the star, the heliocentric wavelength in $\AA$, the heliocentric velocity in $\mathrm{km} \mathrm{s}^{-1}$, the measured equivalent width $W_{\lambda}$ in $\mathrm{m} \AA$, and the corresponding column density $N$ in units of $10^{13} \mathrm{~cm}^{-2}$, listed in Cols. $1-5$, respectively. Columns 6 and 7 contain the average heliocentric $\mathrm{CH}$ velocity and the average column density $\bar{N}$, obtained from the individual measurements weighted by the rotational oscillator strengths.

\section{Analysis}

\subsection{The $C N$ Doppler parameter $b(C N)$ and final $C N$ column densities}

Total CN column densities $N_{\infty}$ obtained from the violet system data in the limit of unsaturated lines are given by $N_{\infty}=\Sigma N_{\infty}\left(N^{\prime \prime}\right)$, where the summation is carried out over rotational levels $N^{\prime \prime}=0,1$ and 2. $N_{\infty}(0)$ and $N_{\infty}(1)$ are taken from Col. 6 of Tables 1 and 2 , and $N_{\infty}(2)$ is calculated from $N_{\infty}(2)=5 \times N_{\infty}(0) \times \mathrm{e}^{-16.325 / T_{\infty}} \cdot T_{\infty}$ is the $\mathrm{CN}$ rotational excitation temperature which is obtained from the population density in $N^{\prime \prime}=0$ and 1 , viz. $T_{\infty}=5.4419 \mathrm{~K} \ln \left(3 \times N_{\infty}(0) / N_{\infty}(1)\right)$. The values of $N_{\infty}$ and $T_{\infty}$ are given in Cols. 2 and 3 of Table 6 , respectively. Uncertainties in $N_{\infty}$ and $T_{\infty}$ resulting from measurement uncertainties in the equivalent widths are given in parentheses. Column densities marked with an asterisk are those where $N_{\infty}(1)$ is not available from the observations, and where $N_{\infty}$ was calculated by assuming a rotational excitation temperature of $T_{\infty}=2.7 \mathrm{~K}$.

The absorption lines of the $\mathrm{CN}(0,0)$ violet system are saturated in general (cf. Gredel et al. 1991), which implies that the values $N_{\infty}$ are only lower limits to the total CN column densities. A curve of growth (COG) analysis is needed to obtain saturation corrections. The method requires knowledge of the micro- and macro-turbulent velocity dispersions, which may be described in terms of Doppler parameters $b$ and the general velocity structure of the line of sight. If the spectral lines are resolved, a generally robust method to infer column densities is to characterise the macroscopic velocity structure in terms of absorption components, and to calculate for each component the column density which corresponds to its microscopic velocity dispersion given by its Doppler $b$-value. For resolved lines, $b$ may be determined from the full width at half maximum of the absorption component. The Doppler parameters $b$ may be different for the various components. Jenkins (1986) demonstrated that this approach does produce sensible results for total column densities. A detailed discussion of the analysis of moderately saturated lines and the effects of the velocity structure is also given by Black \& van Dishoeck (1988).

Our spectral resolution is too coarse to resolve the CN line profile, and indirect methods are applied to infer Doppler parameters $b(\mathrm{CN})$. In cases where unresolved velocity structure is present, the indirect methods provide an effective Doppler parameter which is generally larger than the Doppler parameters of the individual components. While the total CN column densities inferred from e.g. the comparison of unsaturated and saturated absorption lines which arise from a common rotational level are rather robust, the derived effective Doppler paramters may not properly describe the microscopic velocity dispersion. This point is further discussed in Sect. 3.1.4. 

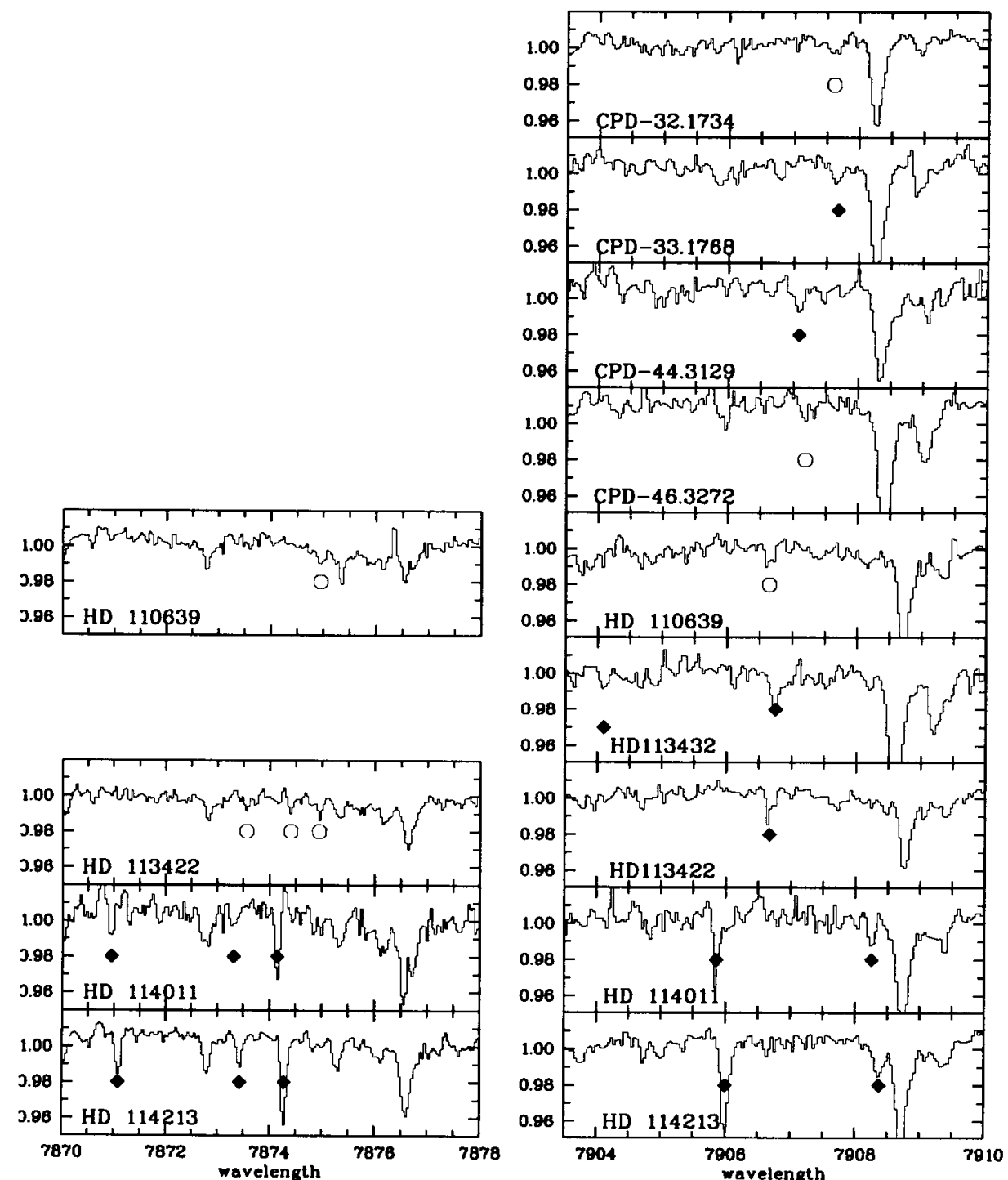

Fig. 4. Stellar spectra covering the $(2,0)$ band of the $\mathrm{CN} \mathrm{A}{ }^{2} \Pi-\mathrm{X}^{2} \Sigma^{+}$red system. Detected CN absorption lines are marked by the filled symbol. Open symbols indicate marginal detections or spectral features which are used to infer upper limits on the CN column density.

\subsubsection{The doublet ratio method}

The doublet ratio method (Strömgren 1948; Münch 1968) compares the strengths of two absorption lines arising from the same rotational level but having different oscillator strengths. If saturation effects prevail, the column density inferred from each line is different. A COG analysis with an iteration on $b$ is performed until the column densities agree. Here we compare the strength of the $R(1)$ and $P(1)$ absorption lines in the $(0,0)$ band of the $\mathrm{CN}$ violet system, both of which arise from the $N^{\prime \prime}=1$ rotational level. The doublet ratio $D R$ is given by $D R=$ $W_{\lambda}(R(1)) / W_{\lambda}(P(1))$. In the limit of unsaturated lines and the absence of measurement errors, the doublet ratio is determined by the ratio of the product of oscillator strengths and rest wavelengths of the two absorption lines, which in the present case is $D R=1.9973$. If both lines are heavily saturated, the doublet ratio approaches the value of $\approx 1$. The difference in the oscillator strengths and wavelengths between the two lines is rather small, however, and very accurate equivalent widths are required for a reliable determination of $b$.

We follow Gredel et al. (1991) and treat the individual spin rotation components explicitly in the COG analysis. A large table of $W_{\lambda}$ and $b$ is computed for the $R(0), R(1)$, and $P(1)$ lines of the $(0,0)$ band of the $\mathrm{CN}$ violet system. Column densities are obtained by cubic spline interpolation within this table. The results of the analysis are given in Table 6, with the CN Doppler parameter $b_{D R}$ which is inferred from the doublet ratio listed in Col. 4. The corresponding total CN column density $N_{D R}$ is given in Col. 5, and the $\mathrm{CN}$ excitation temperature $T_{D R}$ obtained from $N_{D R}(0)$ and $N_{D R}(1)$ is presented in Col. 6 .

Accurate determinations of $b_{\mathrm{DR}}$ are obtained for HD 114011 and HD 114213(-20), with values of 0.98 and $0.75 \mathrm{~km} \mathrm{~s}^{-1}$, respectively. The value in parenthesis added to the star-number indicates the radial velocity of the $\mathrm{CN}$ absorption component in cases where more than 1 component appears. The uncertainties in measured equivalent 

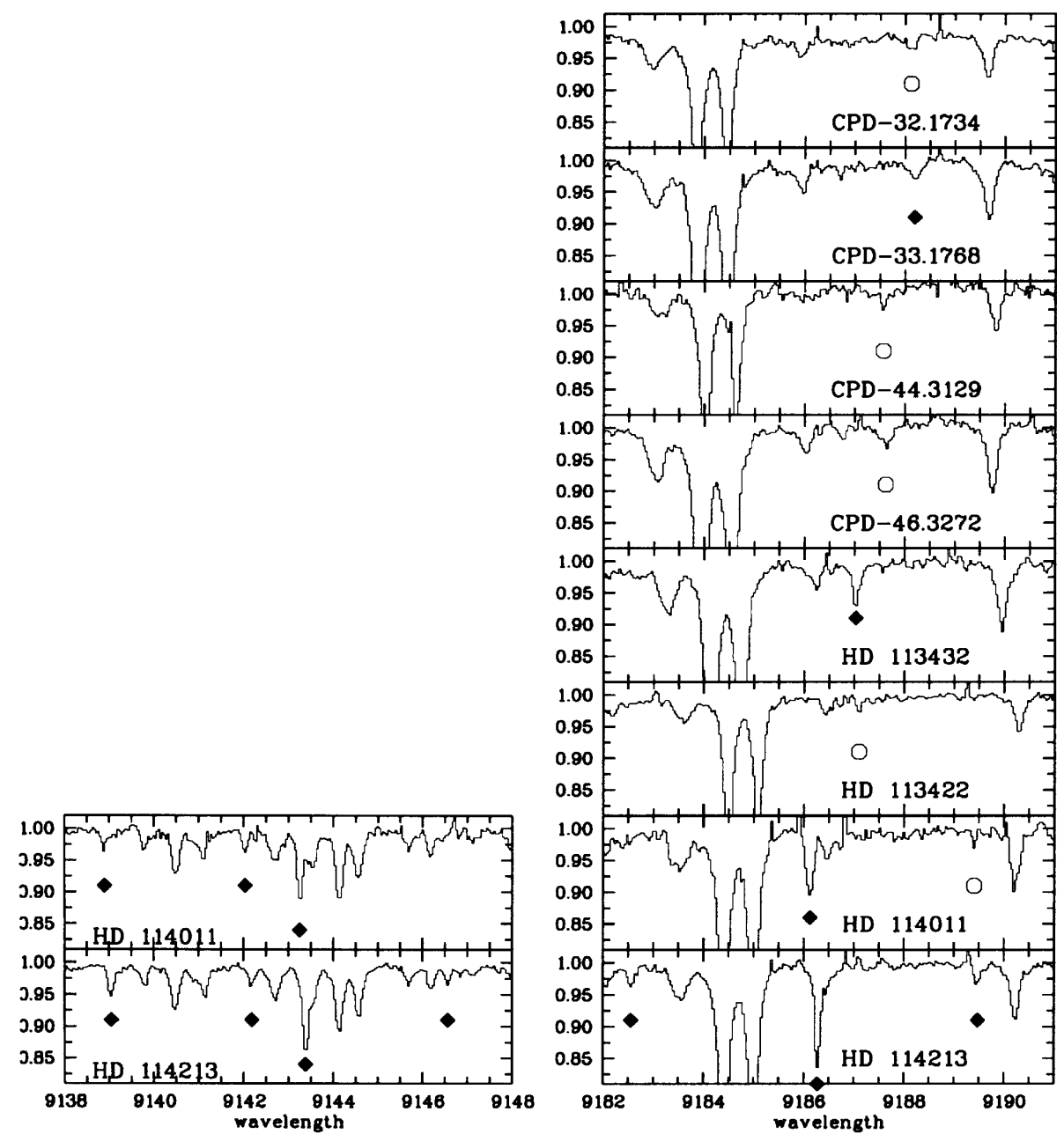

Fig. 5. Same as Fig. 4 for stellar spectra covering the $(1,0)$ band of the CN red system.

widths allow for ranges in $b$ of $0.6-2.3 \mathrm{~km} \mathrm{~s}^{-1}$ toward HD 114011 and $0.5-1.25 \mathrm{~km} \mathrm{~s}^{-1}$ toward HD $114213(-20)$. Resulting ranges in total $\mathrm{CN}$ column densities are given in Col. 5 of Table 6 .

The doublet ratios for gas toward HD 63423, HD 78344, and CPD $-45^{\circ} 3218$ imply values for $b_{D R}$ of $1.95,1.75$, and $1.1 \mathrm{~km} \mathrm{~s}^{-1}$, respectively. The uncertainties in the measured equivalent widths allow for ranges of $0<b<\infty$ toward HD 78344 and CPD $-45^{\circ} 3218$, which is too large to infer uncertainties in $N_{D R}$. Toward HD 63423, a lower limit of $b \geq 0.8 \mathrm{~km} \mathrm{~s}^{-1}$ is obtained. The doublet ratio gives $N_{D R}$ of $12.5 \times 10^{12} \mathrm{~cm}^{-2}$ and a range which arises from the uncertainty in $b$ of $(10-17) \times 10^{12} \mathrm{~cm}^{-2}$. These values are adopted as final CN column density in the discussion of Sect. 4.2. Toward HD 78344, we adopt a total $\mathrm{CN}$ column density of $26.7 \times 10^{12} \mathrm{~cm}^{-2}$ and a range of $(22-32) \times 10^{12} \mathrm{~cm}^{-2}$. Final $\mathrm{CN}$ column densities used in Sect. 4.2 are given in bold face in Table 6 .

In a few cases, doublet ratios larger than 2 or smaller than 1 are obtained, which is unphysical. Doublet ratios larger than two toward HD 110639(+3), HD 113432, and HD $113422(+3)$ are caused by measurement errors. A ratio close to 2 suggests that the $\mathrm{CN}$ lines are not, or only weakly, saturated. The CN absorption lines toward HD 110639(+3) and HD 113422(+3) are relatively weak indeed, and saturation effects are not significant even for small $b$-values. A doublet ratio smaller than 1 is obtained toward HD $114213(+3)$, and small values $(<1.5)$ are obtained toward CPD-32 $2^{\circ} 1734$, CPD-33 $1768, \mathrm{CPD}-46^{\circ} 3272$, and HD 112366. Toward HD $114213(+3)$, the absorption lines are very weak, and the measurement uncertainties allow for doublet ratios of $1<D R<2$. Toward the other stars, the small doublet ratios derived from the measurements would indicate that the absorption lines are heavily saturated. For CPD-32 $2^{\circ} 1734$, CPD-33 ${ }^{\circ} 1768$, HD 76556 , CPD $-46^{\circ} 3272$, and HD 112366, Doppler parameters of $b_{D R}<0.2 \mathrm{~km} \mathrm{~s}^{-1}$ are derived, which are very low. Such low values of $b$ would imply extremely large CN column densities, with $N_{D R}>10^{15} \mathrm{~cm}^{-2}$ in general. Moreover, the resulting $\mathrm{CN}$ excitation temperatures were less than $2 \mathrm{~K}$, which is unphysical. The measurement uncertainties in $W_{\lambda}$ allow for a range of $0<b<\infty \mathrm{kms}^{-1}$ toward the latter five stars, too large to infer meaningful values for $N_{D R}$.

The doublet ratio is not applied to the two velocity components toward HD 61827, because the corresponding equivalent widths are obtained from a 2-component Gaussian fit to a partly resolved line profile. The uncertainties in the equivalent widths toward the remaining 
Table 3. Absorption lines in the $(1,0)$ and $(2,0)$ band of the $\mathrm{CN} \mathrm{A}{ }^{2} \Pi-\mathrm{X}^{2} \Sigma^{+}$red system.

\begin{tabular}{|c|c|c|c|c|c|c|c|c|c|c|}
\hline star & band, line & $J^{\prime \prime}$ & $N^{\prime \prime}$ & $\begin{array}{c}\lambda_{\text {hel }} \\
\AA\end{array}$ & $\begin{array}{r}V_{\text {hel }} \\
\mathrm{km} \mathrm{s}^{-1}\end{array}$ & $\begin{array}{l}W_{\lambda} \\
\mathrm{m} \AA\end{array}$ & $\frac{N\left(J^{\prime \prime}, N^{\prime \prime}\right)}{1}$ & $\frac{N(0)}{0^{12} \mathrm{~cm}^{-2}}$ & $N(1)$ & $\begin{array}{r}T_{\text {red }} \\
\mathrm{K}\end{array}$ \\
\hline $\mathrm{CPD}-32^{\circ} 1734$ & $\begin{array}{l}(2,0) R_{1}(0) \\
(1,0) R_{1}(0)\end{array}$ & $\begin{array}{l}\frac{1}{2} \\
\frac{1}{2}\end{array}$ & $\begin{array}{l}0 \\
0\end{array}$ & $\begin{array}{l}7907.615 \\
9188.122\end{array}$ & $\begin{array}{l}38.4 \\
38.7\end{array}$ & $\begin{array}{l}\leq 1.0 \\
\leq 3.5\end{array}$ & $\begin{array}{l}\leq 4.5 \\
\leq 5.9\end{array}$ & $\leq 5.4$ & & $\ldots$ \\
\hline $\mathrm{CPD}-33^{\circ} 1768$ & $\begin{array}{l}(2,0) R_{1}(0) \\
(1,0) R_{1}(0)\end{array}$ & $\begin{array}{l}\frac{1}{2} \\
\frac{1}{2}\end{array}$ & $\begin{array}{l}0 \\
0\end{array}$ & $\begin{array}{l}7907.680 \\
9188.194\end{array}$ & $\begin{array}{l}40.8 \\
41.0\end{array}$ & $\begin{array}{l}1.5(0.5) \\
6.5(1.0)\end{array}$ & $\begin{array}{r}6.8(2.0) \\
11.0(2.0)\end{array}$ & $9.6(2.0)$ & $\ldots$ & $\ldots$ \\
\hline $\mathrm{CPD}-44^{\circ} 3129$ & $\begin{array}{l}(2,0) R_{1}(0) \\
(1,0) R_{1}(0)\end{array}$ & $\begin{array}{l}\frac{1}{2} \\
\frac{1}{2}\end{array}$ & $\begin{array}{l}0 \\
0\end{array}$ & $\begin{array}{l}7907.086 \\
9187.570\end{array}$ & $\begin{array}{l}18.4 \\
20.7\end{array}$ & $\begin{array}{l}\leq 1.5 \\
\leq 3.5\end{array}$ & $\begin{array}{l}\leq 6.8 \\
\leq 5.9\end{array}$ & $\leq 6.5$ & . & $\ldots$ \\
\hline $\mathrm{CPD}-46^{\circ} 3272$ & $\begin{array}{l}(2,0) R_{1}(0) \\
(1,0) R_{1}(0)\end{array}$ & $\begin{array}{l}\frac{1}{2} \\
\frac{1}{2}\end{array}$ & $\begin{array}{l}0 \\
0\end{array}$ & $\begin{array}{l}7907.185 \\
9187.617\end{array}$ & $\begin{array}{l}22.1 \\
22.2\end{array}$ & $\begin{array}{l}\leq 2.0 \\
\leq 7.0\end{array}$ & $\begin{array}{r}\leq 9.0 \\
\leq 11.8\end{array}$ & $\leq 10.8$ & & $\ldots$ \\
\hline HD 110639 & $\begin{array}{c}(2,0)^{R} Q_{21}(0) \\
(2,0) R_{1}(0)\end{array}$ & $\begin{array}{l}\frac{1}{2} \\
\frac{1}{2}\end{array}$ & $\begin{array}{l}0 \\
0\end{array}$ & $\begin{array}{l}7874.952 \\
7906.646\end{array}$ & $\begin{array}{l}4.0 \\
1.7\end{array}$ & $\begin{array}{l}\leq 1.0 \\
\leq 1.5\end{array}$ & $\begin{array}{l}\leq 7.1 \\
\leq 6.8\end{array}$ & $\leq 6.8$ & . & $\ldots$ \\
\hline HD 113432 & $\begin{array}{l}(2,0) R_{1}(0) \\
(1,0) R_{1}(0) \\
(2,0) R_{1}(1)\end{array}$ & $\begin{array}{l}\frac{1}{2} \\
\frac{1}{2} \\
\frac{3}{2}\end{array}$ & $\begin{array}{l}0 \\
0 \\
1\end{array}$ & $\begin{array}{l}7906.748 \\
9187.030 \\
7904.097\end{array}$ & $\begin{array}{l}5.6 \\
3.1 \\
7.6\end{array}$ & $\begin{array}{r}1.5(1.0) \\
10.0(1.0) \\
\leq 1.0\end{array}$ & $\begin{array}{r}6.8(5.0) \\
16.9(2.0) \\
\leq 7.1\end{array}$ & $13.5(3.0)$ & $\leq 10.5$ & 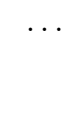 \\
\hline HD 113422 & $\begin{array}{c}(2,0)^{R} Q_{21}(0) \\
(2,0)\left[R_{2}(1)+{ }^{R} Q_{21}(1)\right]\end{array}$ & $\frac{1}{2}+\frac{1}{2}$ & $\begin{array}{l}0 \\
1\end{array}$ & $\begin{array}{l}7874.405 \\
7873.562\end{array}$ & $\begin{array}{l}-16.8 \\
-16.2\end{array}$ & $\leq 1.0$ & $\begin{array}{r}\leq 7.2 \\
\leq 10.5\end{array}$ & $\leq 7.2$ & $\leq 10.5$ & \\
\hline & $\begin{array}{c}(2,0)^{R} Q_{21}(0) \\
(2,0) R_{1}(0) \\
(1,0) R_{1}(0)\end{array}$ & $\begin{array}{l}\frac{1}{2} \\
\frac{1}{2} \\
\frac{1}{2}\end{array}$ & $\begin{array}{l}0 \\
0 \\
0\end{array}$ & $\begin{array}{l}7874.945 \\
7906.667 \\
9187.098\end{array}$ & $\begin{array}{l}3.7 \\
2.5 \\
5.3\end{array}$ & $\begin{array}{l}\leq 1.0 \\
\leq 2.0 \\
\leq 2.5\end{array}$ & $\begin{array}{r}\leq 7.2 \\
\leq 9 \\
\leq 4.2\end{array}$ & $\leq 5.3$ & & \\
\hline HD 114011 & $\begin{array}{c}(2,0)^{S} R_{21}(0) \\
(2,0)^{R} Q_{21}(0) \\
(2,0) R_{1}(0) \\
(1,0)^{S} R_{21}(0) \\
(1,0)^{R} Q_{21}(0) \\
(1,0) R_{1}(0) \\
(2,0)\left[R_{2}(1)+{ }^{R} Q_{21}(1)\right] \\
(2,0)\left[{ }^{Q} R_{12}(1)+Q_{1}(1)\right] \\
(1,0)\left[R_{2}(1)+{ }^{R} Q_{21}(1)\right] \\
(1,0)\left[{ }^{Q} R_{12}(1)+Q_{1}(1)\right]\end{array}$ & $\begin{array}{c}\frac{1}{2} \\
\frac{1}{2} \\
\frac{1}{2} \\
\frac{1}{2} \\
\frac{1}{2} \\
\frac{1}{2} \\
\frac{1}{2}+\frac{3}{2} \\
\frac{1}{2}+\frac{3}{2} \\
\frac{1}{2}+\frac{3}{2} \\
\frac{1}{2}+\frac{3}{2}\end{array}$ & $\begin{array}{l}0 \\
0 \\
0 \\
0 \\
0 \\
0 \\
1 \\
1 \\
1 \\
1\end{array}$ & $\begin{array}{l}7870.958 \\
7874.151 \\
7905.856 \\
9138.896 \\
9143.247 \\
9186.126 \\
7873.318 \\
7908.253 \\
9142.036 \\
9189.405\end{array}$ & $\begin{array}{l}-26.0 \\
-26.5 \\
-28.2 \\
-25.6 \\
-26.1 \\
-26.4 \\
-25.4 \\
-26.5 \\
-26.3 \\
-23.1\end{array}$ & $\begin{array}{r}2.0(1.0) \\
4.0(1.0) \\
3.5(1.0) \\
4.5(3.0) \\
12.0(3.0) \\
17.0(5.0) \\
\leq 1.0 \\
2.0(1.0) \\
5.0(3.0) \\
\leq 2.5\end{array}$ & $\begin{array}{r}34.8(17.0) \\
28.7(7.0) \\
15.8(5.0) \\
29.4(19.0) \\
32.4(8.0) \\
28.7(8.0) \\
\leq 10.5 \\
12.3(6.0) \\
19.8(12.0) \\
\leq 7.0\end{array}$ & $27.5(5.0)$ & $13.1(6.5)$ & 3.0 \\
\hline HD 114213 & $\begin{array}{c}(2,0)^{S} R_{21}(0) \\
(2,0)^{R} Q_{21}(0) \\
(2,0) R_{1}(0) \\
(1,0)^{S} R_{21}(0) \\
(1,0)^{R} Q_{21}(0) \\
(1,0) R_{1}(0) \\
(2,0)\left[R_{2}(1)+{ }^{R} Q_{21}(1)\right] \\
(2,0)\left[{ }^{Q} R_{12}(1)+Q_{1}(1)\right] \\
(1,0)\left[R_{2}(1)+{ }^{R} Q_{21}(1)\right] \\
(1,0)\left[Q_{2}(1)+{ }^{Q} P_{21}(1)\right] \\
(1,0) R_{1}(1) \\
(1,0)\left[{ }^{Q} R_{12}(1)+Q_{1}(1)\right]\end{array}$ & $\begin{array}{c}\frac{1}{2} \\
\frac{1}{2} \\
\frac{1}{2} \\
\frac{1}{2} \\
\frac{1}{2} \\
\frac{1}{2} \\
\frac{1}{2}+\frac{3}{2} \\
\frac{1}{2}+\frac{3}{2} \\
\frac{1}{2}+\frac{3}{2} \\
\frac{1}{2}+\frac{3}{2} \\
\frac{3}{2} \\
\frac{1}{2}+\frac{3}{2}\end{array}$ & $\begin{array}{l}0 \\
0 \\
0 \\
0 \\
0 \\
0 \\
1 \\
1 \\
1 \\
1 \\
1 \\
1\end{array}$ & $\begin{array}{l}7871.081 \\
7874.277 \\
7905.996 \\
9139.047 \\
9143.377 \\
9186.277 \\
7873.426 \\
7908.372 \\
9142.186 \\
9146.557 \\
9182.557 \\
9189.476\end{array}$ & $\begin{array}{l}-21.4 \\
-21.7 \\
-22.9 \\
-20.6 \\
-21.8 \\
-21.4 \\
-21.3 \\
-22.0 \\
-21.3 \\
-21.1 \\
-21.5 \\
-20.7\end{array}$ & $\begin{array}{r}3.0(1.0) \\
6.0(1.0) \\
7.0(1.0) \\
7.0(3.0) \\
10.0(3.0) \\
15.0(3.0) \\
2.0(1.0) \\
4.0(2.0) \\
5.0(3.0) \\
5.0(3.0) \\
5.0(2.0) \\
5.0(3.0)\end{array}$ & $\begin{array}{r}52.2(17.0) \\
43.1(7.0) \\
31.5(5.0) \\
45.7(20.0) \\
27.0(8.0) \\
25.4(5.0) \\
20.9(10.0) \\
24.6(12.0) \\
19.8(12.0) \\
27.0(16.0) \\
13.4(5.0) \\
14.0(8.0)\end{array}$ & $31.9(5.0)$ & $21.1(5.0)$ & 3.6 \\
\hline
\end{tabular}

stars are too large to obtain a reliable determination of $b_{\mathrm{DR}}$.

Toward HD 63423, HD 78344, CPD-453218, HD 110639(+3), HD 114011, HD 113432, HD 113422(+3), and HD 114213(-20), rotational excitation temperatures $T_{D R}$ which result from the column densities obtained with $b_{D R}$ are given in Col. 6 of Table 6 . For CPD $-45^{\circ} 3218, T_{D R}$ is $2.0 \mathrm{~K}$ which is too low. The $\mathrm{CN}$ data toward this star is analysed further in Sect. 3.1.4. A low value of $2.3 \mathrm{~K}$ is also obtained toward HD 114011, but the range in $T_{D R}$ allowed by the range in $b_{D R}$ is $1.2-3.5 \mathrm{~K}$. Toward the other stars, the values are $T_{D R}=2.8-5 \mathrm{~K}$. The relatively high values toward HD 63423 and HD 110639(+3) may indicate the presence of unresolved velocity components because saturation affects the ground state lines to a greater extent. This is because the population density in $N^{\prime \prime}=0$ is always larger than in $N^{\prime \prime}=1$ and because the $R(0)$ line of the $(0,0)$ band of the violet system has a larger oscillator strength than the $R(1)$ or $P(1)$ lines. The high values may also arise from measurement errors in the rotational lines of the $(0,0)$ band of the violet system.

\subsubsection{Comparison of data on the $\mathrm{CN}$ violet and red systems}

In a method similar to the doublet ratio method, saturation effects are determined from a comparison of column 
Table 4. Data for the $\mathrm{CH} \mathrm{B}{ }^{2} \Sigma^{-}-\mathrm{X}^{2} \Pi$ system toward CMa OB1 and NGC 2439.

\begin{tabular}{|c|c|c|c|c|c|c|}
\hline $\begin{array}{l}\text { star } \\
\mathrm{HD} / \mathrm{CPD}\end{array}$ & $\begin{array}{c}\lambda_{\text {hel }} \\
\AA\end{array}$ & $\begin{array}{c}V_{\text {hel }} \\
\mathrm{km} \mathrm{s}^{-1}\end{array}$ & $\begin{array}{l}W_{\lambda} \\
\mathrm{m} \AA\end{array}$ & $10^{13} \mathrm{~cm}^{-2}$ & $\begin{array}{r}\overline{V_{\text {hel }}} \\
\mathrm{km} \mathrm{s}^{-1}\end{array}$ & $10^{13} \mathrm{~cm}^{-2}$ \\
\hline 54662 & 3886.870 & 35.5 & $1.5(0.5)$ & $0.7(0.2)$ & 35.5 & $0.7(0.2)$ \\
\hline \multirow[t]{3}{*}{63423} & 3879.301 & 40.7 & $2.5(0.5)$ & $3.5(0.7)$ & & \\
\hline & 3886.927 & 39.9 & $9.0(0.5)$ & $4.2(0.2)$ & & \\
\hline & 3890.747 & 40.8 & $3.5(0.5)$ & $2.4(0.3)$ & 40.3 & $3.5(0.1)$ \\
\hline \multirow[t]{3}{*}{62150} & 3879.241 & 36.0 & $2.0(1.0)$ & $2.8(1.4)$ & & \\
\hline & 3886.902 & 38.0 & $5.0(0.5)$ & $2.3(0.2)$ & & \\
\hline & 3890.726 & 39.2 & $3.0(1.0)$ & $2.1(0.7)$ & 38.0 & $2.3(0.1)$ \\
\hline \multirow[t]{5}{*}{61827} & 3879.255 & 37.1 & $2.0(0.5)$ & $2.8(0.7)$ & & \\
\hline & 3886.879 & 36.2 & $5.0(0.5)$ & $2.3(0.2)$ & & \\
\hline & 3890.686 & 36.1 & $4.0(0.5)$ & $2.8(0.3)$ & 36.3 & $2.6(0.1)$ \\
\hline & 3886.956 & 42.1 & $3.0(0.5)$ & $1.4(0.2)$ & & \\
\hline & 3890.751 & 41.1 & $2.5(0.5)$ & $1.7(0.3)$ & 41.7 & $1.5(0.1)$ \\
\hline \multirow[t]{3}{*}{62844} & 3879.270 & 38.3 & $3.5(0.5)$ & $4.9(0.7)$ & & \\
\hline & 3886.911 & 38.7 & $10.0(0.5)$ & $4.7(0.2)$ & & \\
\hline & 3890.725 & 39.1 & $6.0(0.5)$ & $4.2(0.3)$ & 38.7 & $4.6(0.1)$ \\
\hline$-32^{\circ} 1734$ & 3886.908 & 38.4 & $12.0(2.0)$ & $5.6(0.9)$ & 38.4 & $5.6(0.9)$ \\
\hline \multirow[t]{2}{*}{$-33^{\circ} 1768$} & 3886.902 & 38.0 & $19.0(5.0)$ & $8.9(2.3)$ & & \\
\hline & 3890.730 & 39.5 & $14.0(5.0)$ & $9.8(3.5)$ & 38.6 & $9.2(1.0)$ \\
\hline 78344 & 3890.477 & 20.0 & $8.5(2.0)$ & $5.9(1.4)$ & 20.0 & $5.9(1.4)$ \\
\hline 75149 & 3886.703 & 22.6 & $3.0(1.0)$ & $1.4(0.5)$ & 22.6 & $1.4(0.5)$ \\
\hline \multirow[t]{2}{*}{74194} & 3886.693 & 21.9 & $2.5(0.5)$ & $1.2(0.2)$ & & \\
\hline & 3890.509 & 22.5 & $2.0(0.5)$ & $1.4(0.3)$ & 22.1 & $1.3(0.1)$ \\
\hline \multirow[t]{2}{*}{76556} & 3886.668 & 19.9 & $7.5(1.0)$ & $3.5(0.5)$ & & \\
\hline & 3890.476 & 19.9 & $6.0(1.0)$ & $4.2(0.7)$ & 19.9 & $3.8(0.2)$ \\
\hline \multirow[t]{2}{*}{75211} & 3886.736 & 25.2 & $1.5(0.5)$ & $1.9(1.0)$ & & \\
\hline & 3890.533 & 24.3 & $1.5(0.5)$ & $2.4(1.0)$ & 24.8 & $2.1(1.0)$ \\
\hline \multirow[t]{2}{*}{75860} & 3879.065 & 22.5 & $2.0(1.0)$ & $2.8(1.4)$ & & \\
\hline & 3886.685 & 21.3 & $4.0(1.0)$ & $1.9(0.5)$ & 21.6 & $2.1(0.2)$ \\
\hline \multirow{2}{*}{$-44^{\circ} 3129$} & 3886.684 & 21.2 & $9.0(2.0)$ & $4.2(0.9)$ & & \\
\hline & 3890.493 & 21.2 & $10.0(3.0)$ & $7.0(2.1)$ & 21.2 & $5.3(0.5)$ \\
\hline \multirow[t]{2}{*}{$-46^{\circ} 3272$} & 3886.685 & 21.3 & $12.0(1.0)$ & $5.6(0.5)$ & & \\
\hline & 3890.490 & 21.0 & $7.5(3.0)$ & $5.2(2.1)$ & 21.2 & $5.5(0.4)$ \\
\hline \multirow[t]{3}{*}{$-45^{\circ} 3218$} & 3879.061 & 22.1 & $5.0(1.5)$ & $7.0(2.1)$ & & \\
\hline & 3886.688 & 21.5 & $11.0(1.5)$ & $5.1(0.7)$ & & \\
\hline & 3890.506 & 22.2 & $13.5(1.5)$ & $9.4(1.0)$ & 21.8 & $6.9(0.2)$ \\
\hline
\end{tabular}

densities derived from absorption lines which arise in different electronic bands. For CN, a comparison between the violet and red systems works particularly well, because the difference in oscillator strengths is very large. In the following, we treat the lines in the CN red system as unsaturated, and perform a COG analysis for data involving the violet system with an iteration on $b$ until the column densities agree with those inferred from the red system. The Doppler parameters $b_{\mathrm{VR}}$ obtained in this way are listed in Col. 7 of Table 6 ; total CN column densities $N_{\mathrm{VR}}$ and rotational excitation temperatures $T_{\mathrm{VR}}$ are given in Cols. 8 and 9 , respectively.

Very narrow ranges in $b_{\mathrm{VR}}$ are obtained toward the three stars in Cen OB1 which have accurate detections of absorption lines in the $\mathrm{CN}$ red system. We find $b_{\mathrm{VR}}=0.6-0.8 \mathrm{~km} \mathrm{~s}^{-1}$ toward HD 113432, 1.05$1.25 \mathrm{~km} \mathrm{~s}^{-1}$ toward HD 114011, and $0.65-0.85 \mathrm{~km} \mathrm{~s}^{-1}$ toward HD 114213(-20). The Doppler parameters found for
HD 114011 and HD 114213 agree remarkably well with those obtained from the doublet ratio method. Column densities $N(1)$ calculated for $b_{\mathrm{VR}}$ from the violet system data are $10.8 \times 10^{12} \mathrm{~cm}^{-2}$ and $14.1 \times 10^{12} \mathrm{~cm}^{-2}$ for HD 114011 and HD 114213(-20), respectively; these are adopted in the following as $N(1)$. These values are smaller than the column densities inferred from the red system data, $N(1)=(13.1 \pm 6.5) \times 10^{12} \mathrm{~cm}^{-2}$ and $N(1)=(21.1 \pm 5.0) \times 10^{12} \mathrm{~cm}^{-2}$ toward HD 114011 and HD 114213(-20), respectively, but the corresponding absorption lines in the red system are relatively weak and the measurement uncertainties are large. The total CN column densities derived toward HD 113432, HD 114011, and HD 114213(-20) from the comparison of violet and red system data are adopted as final in the discussion of Sect. 4.2 .

Other sight lines yield useful information with this approach. For CPD- $32^{\circ} 1734, \mathrm{CPD}-44^{\circ} 3129$, and 
Table 5. $\mathrm{CH} \mathrm{B}^{2} \Sigma^{-}-\mathrm{X}{ }^{2} \Pi$ system data toward Cen OB1.

\begin{tabular}{|c|c|c|c|c|c|c|}
\hline $\begin{array}{l}\text { star } \\
\mathrm{HD} / \mathrm{CPD}\end{array}$ & $\lambda_{\text {hel }}$ & $\begin{array}{r}V_{\text {hel }} \\
\mathrm{km} \mathrm{s}^{-1}\end{array}$ & $\begin{array}{l}W_{\lambda} \\
\mathrm{m} \AA\end{array}$ & $10^{13} \mathrm{~cm}^{-2}$ & $\begin{array}{r}\overline{V_{\text {hel }}} \\
\mathrm{km} \mathrm{s}^{-1}\end{array}$ & $\begin{array}{r}\bar{N} \\
10^{13} \mathrm{~cm}^{-2}\end{array}$ \\
\hline 111904 & 3886.219 & -14.6 & $1.0(0.5)$ & $0.5(0.2)$ & -14.6 & $0.5(0.2)$ \\
\hline 111973 & 3886.503 & 7.2 & $1.0(0.5)$ & $0.5(0.2)$ & 7.2 & $0.5(0.2)$ \\
\hline \multirow[t]{2}{*}{$-59^{\circ} 4551$} & 3886.252 & -12.1 & $1.5(0.5)$ & $0.7(0.2)$ & -12.1 & $0.7(0.2)$ \\
\hline & 3886.465 & 4.3 & $1.5(0.5)$ & $0.7(0.2)$ & 4.3 & $0.7(0.2)$ \\
\hline 114886 & 3886.465 & 4.3 & $1.0(0.5)$ & $0.5(0.2)$ & 4.3 & $0.5(0.2)$ \\
\hline \multirow[t]{3}{*}{112366} & 3878.841 & 5.2 & $3.0(1.0)$ & $4.2(1.4)$ & & \\
\hline & 3886.478 & 5.3 & $8.0(1.0)$ & $3.7(0.5)$ & & \\
\hline & 3890.292 & 5.8 & $5.0(1.0)$ & $3.5(0.7)$ & 5.4 & $3.7(0.1)$ \\
\hline 115363 & 3886.454 & 3.5 & $5.0(1.0)$ & $2.3(0.5)$ & 3.5 & $2.3(0.5)$ \\
\hline \multirow[t]{6}{*}{110639} & 3878.512 & -20.2 & $2.0(1.0)$ & $2.8(1.4)$ & & \\
\hline & 3886.137 & -20.9 & $3.5(1.0)$ & $1.6(0.5)$ & & \\
\hline & 3889.943 & -21.1 & $2.0(0.5)$ & $1.4(0.3)$ & -20.9 & $1.8(0.1)$ \\
\hline & 3878.810 & 2.8 & $1.5(1.0)$ & $2.1(1.4)$ & & \\
\hline & 3886.459 & 3.9 & $2.5(1.0)$ & $1.2(0.5)$ & & \\
\hline & 3890.247 & 2.3 & $1.5(0.5)$ & $1.0(0.3)$ & 3.2 & $1.3(0.1)$ \\
\hline \multirow[t]{3}{*}{114011} & 3878.430 & -26.5 & $5.0(3.0)$ & $7.0(4.2)$ & & \\
\hline & 3886.084 & -25.0 & $10.0(3.0)$ & $4.7(1.4)$ & & \\
\hline & 3889.886 & -25.5 & $5.0(3.0)$ & $3.5(2.1)$ & -25.4 & $4.7(0.4)$ \\
\hline \multirow[t]{4}{*}{113432} & 3886.180 & -17.6 & $2.0(1.0)$ & $0.9(0.5)$ & -17.6 & $0.9(0.5)$ \\
\hline & 3878.847 & 5.6 & $3.0(1.5)$ & $4.2(2.1)$ & & \\
\hline & 3890.277 & 4.6 & $4.5(1.0)$ & $3.1(0.7)$ & & \\
\hline & 3886.469 & 4.6 & $8.0(1.0)$ & $3.7(0.5)$ & 4.8 & $3.5(0.5)$ \\
\hline \multirow[t]{4}{*}{113422} & 3886.173 & -18.2 & $5.5(1.5)$ & $2.6(0.7)$ & & \\
\hline & 3889.980 & -18.2 & $3.0(1.0)$ & $2.1(0.7)$ & -18.2 & $2.4(0.3)$ \\
\hline & 3886.455 & 3.5 & $2.5(1.0)$ & $1.2(0.5)$ & & \\
\hline & 3890.264 & 3.6 & $2.5(1.0)$ & $1.7(0.7)$ & 3.6 & $1.4(0.2)$ \\
\hline \multirow[t]{6}{*}{112272} & 3878.649 & -9.6 & $2.5(1.5)$ & $3.5(2.1)$ & & \\
\hline & 3886.302 & -8.2 & $6.0(0.5)$ & $2.8(0.2)$ & & \\
\hline & 3890.111 & -8.2 & $4.0(0.5)$ & $2.8(0.3)$ & -8.4 & $2.9(0.1)$ \\
\hline & 3878.825 & 3.9 & $1.5(1.5)$ & $2.1(2.1)$ & & \\
\hline & 3886.462 & 4.1 & $5.5(0.5)$ & $2.6(0.2)$ & & \\
\hline & 3890.268 & 3.9 & $4.5(0.5)$ & $3.1(0.3)$ & 4.0 & $2.7(0.1)$ \\
\hline \multirow[t]{4}{*}{114213} & 3886.149 & -20.0 & $7.0(1.5)$ & $3.3(0.7)$ & & \\
\hline & 3889.967 & -19.2 & $8.5(2.0)$ & $5.9(1.4)$ & -19.7 & $4.3(0.4)$ \\
\hline & 3886.428 & 1.5 & $4.0(1.0)$ & $1.9(0.5)$ & & \\
\hline & 3890.256 & 3.0 & $4.5(2.0)$ & $3.1(1.4)$ & 2.1 & $2.4(0.3)$ \\
\hline
\end{tabular}

CPD-46 3272 , the upper limits in $N^{\prime \prime}=0$ obtained from the $R_{1}(0)$ lines in the $(1,0)$ and $(2,0)$ bands of the $\mathrm{CN}$ red system (cf. Table 3 ) indicate lower limits in $b_{V R}$ of 2 , 0.75 , and $3 \mathrm{kms}^{-1}$, respectively. The values $N_{V R}$ given in Col. 8 of Table 6 are column densities obtained for the lowest Doppler parameters $b_{V R}$. These values may be considered as upper limits on the total $\mathrm{CN}$ column density. Toward CPD $-46^{\circ} 3272$, we suggest a final CN column density of $(14 \pm 1) \times 10^{12} \mathrm{~cm}^{-2}$. For CPD-33 1768 , the column density $N(0)$ inferred from the violet system is brought into agreement with results on the red system for a Doppler parameter of $b_{V R}=1.75 \mathrm{~km} \mathrm{~s}^{-1}$, but a range of $1.25 \mathrm{~km} \mathrm{~s}^{-1}<b<\infty \mathrm{km} \mathrm{s}^{-1}$ is allowed by the uncertainties in the equivalent widths of the $R(0)$ line in the violet system.

Rotational excitation temperatures $T_{V R}$ inferred from the population distributions in $N^{\prime \prime}=0$ and $N^{\prime \prime}=1$ are listed in Col. 9 of Table 6. Calculated temperatures are in the range of 2.5-3.2 K. Toward HD 114011, HD 113432, and HD $114213(+3)$, uncertainties in $T_{\mathrm{VR}}$ of $0.2-0.3 \mathrm{~K}$ are given in parentheses. Toward the other stars, uncertainties are significantly larger.

\subsubsection{Comparison with data on $\mathrm{CO}$ emission lines}

The width of millimetre CO emission lines may be used to estimate $b(\mathrm{CN})$. Such observations resolve the CO line profiles in general, and the CO Doppler parameter $b_{\mathrm{CO}}$ is obtained from the full width at half maximum $\Delta V$ : $b_{\mathrm{CO}}=\Delta V / 1.665$. We observed CO millimetre emission using the Swedish-ESO SEST telescope on La Silla, with a velocity resolution of $0.14 \mathrm{kms}^{-1}$ at the frequency of the $J=1 \rightarrow 0$ CO line. A complete description of the $\mathrm{CO}$ data will be given elsewhere. Here we proceed to 
Table 6. Summary of CN analysis ${ }^{a}$.

\begin{tabular}{|c|c|c|c|c|c|c|c|c|c|c|c|c|c|}
\hline $\begin{array}{l}\text { star } \\
\mathrm{HD} / \mathrm{CPD}\end{array}$ & $N_{\infty}$ & $T_{\infty}$ & $b_{D R}$ & $N_{D R}$ & $T_{D R}$ & $b_{V R}$ & $N_{V R}$ & $T_{V R}$ & $b_{\mathrm{CO}}^{\dagger}$ & $N_{b(\mathrm{CO})}$ & $T_{b(\mathrm{CO})}$ & $N_{b=1}$ & $T_{b=1}$ \\
\hline 63423 & $10.9(0.2)$ & $5.2(0.3)$ & $1.95_{-1.2}^{+\infty}$ & $12.5_{-2.5}^{+4.5}$ & 4.8 & & & & $c$ & & & $14.7(1.0)$ & $4.2(0.3)$ \\
\hline 62150 & $0.4(0.3)^{*}$ & $(2.7)$ & & & & & & & $n$ & & & $0.5(0.3)^{*}$ & $(2.7)$ \\
\hline $61827(+35)$ & $3.1(0.4)$ & $2.7(0.7)$ & & & & & & & $c$ & & & $3.6(0.7)$ & $2.6(0.9)$ \\
\hline $61827(+41)$ & $3.2(0.4)$ & $2.7(0.7)$ & & & & & & & $c$ & & & $3.6(0.7)$ & $2.6(0.9)$ \\
\hline 62844 & $2.8(0.4)$ & $3.3(0.9)$ & & & & & & & $n$ & & & $3.1(0.6)$ & $3.1(1.0)$ \\
\hline$-32^{\circ} 1734$ & $7.3(0.6)$ & $3.3(0.6)$ & $<0.1$ & - & & $\geq 2.0$ & $\leq 8.5$ & 3.2 & $c$ & & & $10.0(2.0)$ & $2.9(0.8)$ \\
\hline$-33^{\circ} 1768$ & $9.5(1.2)$ & $2.8(0.7)$ & $<0.1$ & - & & $1.75_{-0.5}^{+\infty}$ & 12.4 & 2.5 & $c$ & & & $17.1_{-4.5}^{+6.6}$ & $2.1(0.6)$ \\
\hline 78344 & $17.5(1.2)$ & $3.6(0.5)$ & 1.75 & $26.7(5.0)$ & 2.9 & & & & 1.0 & 77.3 & 1.8 & $77.3_{-23}^{+46}$ & $1.8(0.4)$ \\
\hline 75149 & $0.6(0.2)^{*}$ & $(2.7)$ & & & & & & & $c$ & & & $0.7(0.2)^{*}$ & $(2.7)$ \\
\hline 76556 & $8.3(0.4)$ & $3.3(0.3)$ & $<0.1$ & - & & & & & 0.7 & 15.5 & 2.4 & $11.8_{-1.4}^{+1.1}$ & $2.8(0.4)$ \\
\hline 75211 & $3.3(0.4)$ & $2.4(0.7)$ & & & & & & & $c$ & & & $4.0(0.5)$ & $2.1(0.6)$ \\
\hline$-44^{\circ} 3129$ & $5.6(0.6)^{*}$ & $(2.7)$ & & & & $\geq 0.75$ & $\leq 9.1^{*}$ & $(2.7)$ & $c$ & & & $7.8(1.0)^{*}$ & $(2.7)$ \\
\hline$-46^{\circ} 3272$ & $12.6(1.0)$ & $2.9(0.4)$ & $<0.1$ & - & & $\geq 3.0$ & $\leq 14.8$ & 2.7 & $c$ & & & $35.2(8.0)$ & $1.8(0.3)$ \\
\hline$-45^{\circ} 3218$ & $20.8(1.2)$ & $4.2(0.6)$ & 1.1 & 80.1 & 2.0 & & & & $c$ & & & $115_{-36}^{+65}$ & $1.8(0.4)$ \\
\hline 112366 & 12.1(1.0) & $6.7_{-1.6}^{+2.6}$ & $<0.2$ & - & & & & & 0.7 & $19.0(2.0)$ & 5.1 & $16.3_{-4.5}^{+0.7}$ & $5.8_{-1.6}^{+3.5}$ \\
\hline 115363 & $2.8(0.2)$ & $2.4(0.4)$ & & & & & & & 1.6 & 3.0 & 2.4 & $3.1(0.4)$ & $2.3(0.3)$ \\
\hline $110639(-20)$ & $1.5(0.2)$ & $3.0(1.0)$ & & & & $\leq 0.1$ & & & 0.55 & $1.6(0.5)$ & 2.8 & $1.6(0.4)$ & $2.9(1.0)$ \\
\hline $110639(+3)$ & $4.5(0.2)$ & $4.5(0.5)$ & $\infty$ & 4.2 & 4.0 & & & & 0.55 & $5.7(0.5)$ & 3.3 & $5.0(0.5)$ & $3.6(0.5)$ \\
\hline 114011 & $18.0(0.4)$ & $4.3(0.3)$ & $0.98_{-0.4}^{+1.3}$ & $53.9_{-34}^{+800}$ & $2.3(1.2)$ & $1.15(0.1)$ & $37.9(4.0)$ & $2.7(0.2)$ & 1.4 & 29.8 & 3.1 & $51.4_{-8}^{+3}$ & $2.3(0.2)$ \\
\hline 113432 & $9.6(0.4)$ & $3.8(0.4)$ & $\infty$ & 9.2 & 3.8 & $0.7(0.1)$ & $18.8(3.0)$ & $2.5(0.3)$ & 0.5 & 43.1 & 1.8 & $13.8(1.0)$ & $3.0(0.4)$ \\
\hline $113422(-18)$ & $2.9(0.4)$ & $4.7_{-1.2}^{+2.3}$ & & & & $\leq 0.1$ & & & 1.9 & 3.0 & 3.6 & $3.2(0.5)$ & $3.6(1.0)$ \\
\hline $113422(+3)$ & $4.0(0.4)$ & $3.6(1.0)$ & $\infty$ & 3.8 & 3.6 & $\leq 0.1$ & & & 0.85 & 4.5 & 3.2 & $4.4(1.0)$ & $3.2(1.2)$ \\
\hline 112272 & $1.8(0.4)$ & $2.6(0.6)$ & & & & & & & $c$ & & & $1.8(0.4)$ & $2.6(0.6)$ \\
\hline $114213(-20)$ & $17.0(0.4)$ & $5.6(0.5)$ & $0.75_{-0.3}^{+0.5}$ & $46.8_{-22}^{+450}$ & $2.8(1.4)$ & $0.75(0.1)$ & $46.8(10.0)$ & $2.8(0.3)$ & 1.15 & 26.3 & 4.2 & $29.4_{-3}^{+0.6}$ & $3.8(0.4)$ \\
\hline $114213(+3)$ & $2.6(0.4)$ & $2.8_{-0.8}^{+1.2}$ & $<0.1$ & - & & & & & 0.7 & 3.0 & 2.6 & $2.8(0.8)$ & $2.7(1.0)$ \\
\hline
\end{tabular}

${ }^{a} N$ in $10^{12} \mathrm{~cm}^{-2}, b$ in $\mathrm{km} \mathrm{s}^{-1}, T$ in $\mathrm{K}$.

Values in bold face are CN column densities adopted in the analysis of Sect. 4.2.

${ }^{\dagger} c$ identifies complex CO line profiles. $n$ identifies lines of sight where $\mathrm{CO}$ is not detected.

${ }^{*}$ Derived from $N(0)$ and $T_{\mathrm{ex}}=2.7 \mathrm{~K}$. 
calculate CN column densities $N_{b(\mathrm{CO})}$ using $b_{\mathrm{CO}}$ as the Doppler parameter for $\mathrm{CN}$. The main disadvantage of this method is that emission from material behind the star may not easily be discriminated from the foreground material which is seen in absorption. For complex CO line profiles and for $\mathrm{CO}$ emission which is spread over a large range of radial velocities this uncertainty becomes prominent. In cases where the $\mathrm{CO}$ emission is narrow and appears at the velocity of the $\mathrm{CN}$ absorption lines, it is likely but not certain that $\mathrm{CO}$ and $\mathrm{CN}$ occupy the same parcels of gas. The velocity dispersions may be different anyway. Bucher \& Glinski (1999) showed that care should be exercised when analysing the line profiles of diatomic molecules. In particular, the line profiles of molecules such as $\mathrm{CH}^{+}$and $\mathrm{CH}$ reflect the non-Boltzmann translational energy distribution at which the molecules are formed. This is because the random velocities of these molecules which result from $\mathrm{H}$ and $\mathrm{H}_{2}$ reactions are not thermalised by collisions, and line profiles substantially broader than what is expected from a Boltzmann distribution at the kinetic temperature may result. The case is particularly pronounced for $\mathrm{CH}^{+}$, which is removed upon every collision with $\mathrm{H}_{2}$, and for $\mathrm{CH}$ to a lesser extent. For $\mathrm{CN}$ and $\mathrm{CO}$, the effect is probably less pronounced as neither molecule is formed via reactions involving $\mathrm{H}$ and $\mathrm{H}_{2}$ at the low temperatures which prevail in quiescent translucent clouds.

In Col. 10 of Table 6 we list the CO Doppler parameter $b_{\mathrm{CO}}$ obtained from those $\mathrm{CO}$ emission lines where the CO emission occurs in single Gaussians near the velocity of CN absorption. CO line profiles which are broad and complex and where the radial velocity of the emission is spread over many $\mathrm{km} \mathrm{s}^{-1}$ are labelled with $c$. For those lines of sight, a de-composition of the $\mathrm{CO}$ line profile into multiple Gaussians is arbitrary. Lines of sight where $\mathrm{CO}$ is not detected, with $\mathrm{CO}$ antenna temperatures of $T_{\mathrm{A}}^{*}<0.1 \mathrm{~K}$, are labelled with $n$. The resulting $\mathrm{CN}$ column densities $N_{b(\mathrm{CO})}$ and $\mathrm{CN}$ rotational excitation temperatures $T_{b(\mathrm{CO})}$ are presented in Cols. 11 and 12 , respectively. Toward CPD-33ำ 1768 , HD 78344, HD 76556, $\mathrm{CPD}-44^{\circ} 3129, \mathrm{CPD}-45^{\circ} 3218$, and HD 115363 , the rotational excitation temperatures calculated for $b_{\mathrm{CO}}$ are very low, suggesting the presence of unresolved velocity structure or of measurement errors in the equivalent widths. For the other stars, the inferred CN column densities are consistent with those inferred from the other methods, except toward HD 113432 where $N_{b(\mathrm{CO})}$ is very large. These findings provide additional confidence that the $\mathrm{CN}$ saturation corrections are realistic. Toward stars HD 112366 , HD $110639(+3)$, and HD $110639(-20)$, we adopt $N_{b(\mathrm{CO})}$ as final values.

\subsubsection{Other methods and final CN column densities}

For star $\mathrm{CPD}-45^{\circ} 3218$, the doublet ratio yields a value for $b_{D R}$ of $\approx 1 \mathrm{~km} \mathrm{~s}^{-1}$, but the corresponding CN excitation temperature is $T_{D R}=2.0 \mathrm{~K}$ which is too low. The measurement errors are relatively low. Instead, we iterate on $b$ until we obtain a $\mathrm{CN}$ excitation temperature which is close to $T_{\mathrm{CMB}}$. We obtain $2.7 \mathrm{~K}$ for a Doppler value of $1.4 \mathrm{~km} \mathrm{~s}^{-1}$, yielding a total $\mathrm{CN}$ column density of $42 \times 10^{12} \mathrm{~cm}^{-2}$. For $b=1.5 \mathrm{~km} \mathrm{~s}^{-1}$, $N=38 \times 10^{12} \mathrm{~cm}^{-2}$ and $T=2.86 \mathrm{~K}$. We suggest a value of $N=(40 \pm 10) \times 10^{12} \mathrm{~cm}^{-2}$ as the total $\mathrm{CN}$ column density toward CPD $-45^{\circ} 3218$.

Toward CPD- $33^{\circ} 1768$, the rotational excitation temperature is $2.8 \mathrm{~K}$ and decreases significantly for any saturation correction applied. As noted above, the decrease in the excitation temperature with decreasing Doppler parameter arises from the fact that $N(0)$ is more susceptible to optical depth effects. Toward CPD- $33^{\circ} 1768$, we use $N_{\infty}$ as the final CN column density in the discussion of Sect. 4.2.

Toward HD 75211 and HD 115363, the CN excitation temperatures obtained from $N_{\infty}(0)$ and $N_{\infty}(1)$ are $2.4 \mathrm{~K}$. Such low, and unphysical, values may result from measurement errors in the equivalent widths of the $R(0), R(1)$, and $P(1)$ lines. In both cases, the absorption lines are relatively weak, and saturation corrections are not large. We adopt $N_{\infty}$ as final CN column densities. For a few other stars, measured equivalent widths are small as well, and saturation effects are not significant even for Doppler parameters of $b \approx 0.5 \mathrm{~km} \mathrm{~s}^{-1}$. Such is the case for HD 62150 and HD 75149, where we adopt the column densities obtained for a Doppler parameter of $1 \mathrm{~km} \mathrm{~s}^{-1}$ as final.

If all other methods fail and if there is no information whatsoever concerning the $\mathrm{CN} b$-value, we adopt an ad hoc value of $b=1.0 \mathrm{~km} \mathrm{~s}^{-1}$ to infer $\mathrm{CN}$ column densities. The relatively reliable determinations of $b_{D R}$ and $b_{V R}$ toward HD 114011 and HD 114213(-20) suggest values of the order of $1 \mathrm{~km} \mathrm{~s}^{-1}$. The ultra-high resolution observations of Lambert et al. (1990), Crawford (1995), and Crawford (1997) toward $\zeta$ Oph, HD 169454, and two stars in the Scorpius-Centaurus association indicate $b(\mathrm{CN})=0.3-0.7 \mathrm{~km} \mathrm{~s}^{-1}$ for single and resolved velocity components. We also note that high-resolution observations of a sample of some 20 stars in the Cep OB2 and Cep OB3 associations indicate CN Doppler parameters of $1 \mathrm{~km} \mathrm{~s}^{-1}$ (Pan \& Federman, unpublished). For completeness, we list total CN column densities $N_{b=1}$ for all stars and corresponding excitation temperatures $T_{b=1}$ obtained with $b=1.0 \mathrm{~km} \mathrm{~s}^{-1}$ in Cols. 13 and 14 of Table 6 , respectively. Uncertainties in $N_{b=1}$ and $T_{b=1}$ allowed by the uncertainties in the measured equivalent widths are given in parentheses. Values for $N_{b=1}$ derived toward $\mathrm{CPD}-32^{\circ} 1734$, HD $76556, \mathrm{CPD}-44^{\circ} 3129$, HD $113422(-18)$, and HD $113422(+3)$ are adopted as final in Sect. 4.2.

An effective Doppler parameter of $b=1 \mathrm{~km} \mathrm{~s}^{-1}$ may be used for moderately saturated lines which are composed of 2-3 absorption components separated by $1-2 \mathrm{~km} \mathrm{~s}^{-1}$, if the total column density is $N\left(N^{\prime \prime}=0\right)<10^{13} \mathrm{~cm}^{-2}$ and if the $b=0.5-0.7 \mathrm{~km} \mathrm{~s}^{-1}$ for each of the absorption components. For instance, we obtain for HD 76556 a total column density in $N^{\prime \prime}=0$ of $N\left(N^{\prime \prime}=0\right)=11.1 \times 10^{12} \mathrm{~cm}^{-2}$ from the measured equivalent width of $23 \mathrm{~m} \AA$ in the $R(0)$ 
line of the CN blue system, if we assume that the line is formed from three velocity components separated by $2 \mathrm{~km} \mathrm{~s}^{-1}$ from each other, each component characterised by $b=0.7 \mathrm{~km} \mathrm{~s}^{-1}$. A single component with an effective Doppler parameter of $b=1 \mathrm{~km} \mathrm{~s}^{-1}$ yields $N\left(N^{\prime \prime}=0\right)=$ $8.3 \times 10^{12} \mathrm{~cm}^{-2}$. The column obtained in the limit for unsaturated lines is $N_{\infty}\left(N^{\prime \prime}=0\right)=5.1 \times 10^{12} \mathrm{~cm}^{-2}$.

For HD $61827(+35)$, HD $61827(+41)$, HD 62844 , HD 112272, and HD $114213(+3)$, the CN excitation temperature inferred in the limit of $b \rightarrow \infty$ is $2.7 \mathrm{~K}$ and slightly smaller for $b=1 \mathrm{~km} \mathrm{~s}^{-1}$. In all cases, the absorption lines are relatively weak and saturation corrections for Doppler parameters $b>0.5 \mathrm{~km} \mathrm{~s}^{-1}$ are small. For these clouds, we adopt the $\mathrm{CN}$ column densities inferred from $b=1 \mathrm{~km} \mathrm{~s}^{-1}$ as final.

\subsection{Comparison of data on the $C H A^{2} \Delta-X^{2} \Pi$ and $B^{2} \Sigma^{-}-X^{2} \Pi$ systems}

The observations of $\mathrm{CH}$ absorption lines in the $(0,0)$ band of the $\mathrm{CH} \mathrm{B}-\mathrm{X}$ system presented above provide an independent and useful check on the final $\mathrm{CH}$ column densities of Gredel (1997). Absorption from the $(0,0)$ band of the $\mathrm{CH} \mathrm{A}{ }^{2} \Delta-\mathrm{X}{ }^{2} \Pi$ system toward the stars observed here was corrected for saturation effects using a $\mathrm{CH}$ Doppler parameter $b(\mathrm{CH})$ of $1.5 \mathrm{~km} \mathrm{~s}^{-1}$ in general. The particular value is suggested by high-resolution observations of interstellar CH where the line profile has been resolved (e.g., Crane et al. 1995, Crawford 1995). In a few cases, the saturation corrections of Gredel (1997) are substantial and exceed values of $\left(N_{b}(\mathrm{CH})-N_{\infty}(\mathrm{CH})\right) / N_{\infty}(\mathrm{CH}) \geq 2$.

The present data for HD 54662 in CMa OB1 are very consistent with the earlier results of Paper I. The saturation corrections obtained for $b(\mathrm{CH})$ of $1.5 \mathrm{~km} \mathrm{~s}^{-1}$ are small and do not exceed $10 \%$. For the stars in CMa OB1, the values $N_{b}(\mathrm{CH})$ as listed in Paper I are adopted as final $\mathrm{CH}$ column densities in the discussion of Sects. 4.2 and 4.3.

Toward HD 63423 and HD 62150, the present $\mathrm{CH}$ measurements agree very well with the COG analysis of Paper I. Toward HD 61827, HD 62844, and $\mathrm{CPD}-33^{\circ} 1768$, the $\mathrm{CH}$ column densities inferred here are larger than $N_{\infty}(\mathrm{CH})$ but lower than $N_{b}(\mathrm{CH})$ of Paper I. This suggests $\mathrm{CH}$ Doppler parameters greater than $1.5 \mathrm{~km} \mathrm{~s}^{-1}$. For the three stars, we obtain final $\mathrm{CH}$ column densities which are lower by some $20-25 \%$ compared to the values for $N_{b}(\mathrm{CH})$ given in Paper I. Toward CPD $-32^{\circ} 1734$, the $\mathrm{CH}$ column density obtained here is lower than $N_{\infty}(\mathrm{CH})$ inferred from both the CAT and the EMMI measurements of Paper I. The present measurement is associated with a relatively large error of some $15 \%$. We adopt a final column density of $N(\mathrm{CH})=9 \times 10^{13} \mathrm{~cm}^{-2}$ for CPD-32 ${ }^{\circ} 1734$.

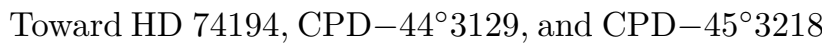
in Vela OB1, CH column densities inferred here agree with those of Paper I obtained with $b(\mathrm{CH})=1.5 \mathrm{~km} \mathrm{~s}^{-1}$. Toward HD 75149, HD 75211, HD 76556, and $\mathrm{CPD}-46^{\circ} 3272$, the $\mathrm{CH}$ column densities are some $10 \%$ lower than those of Paper I. Toward HD 75860, we adopt a value of $N(\mathrm{CH})=2.8 \times 10^{13} \mathrm{~cm}^{-2}$ which is some $20 \%$ lower than that given in Paper $\mathrm{I}$, and $N(\mathrm{CH})=$ $6 \times 10^{13} \mathrm{~cm}^{-2}$ for HD 78344. The $\mathrm{CH}$ column densities toward HD 114886, HD 112366, HD 115363, HD 112272, and HD 114213 in Cen OB1 derived here agree with those obtained with $b(\mathrm{CH})=1.5 \mathrm{~km} \mathrm{~s}^{-1}$. Toward HD 110639 , HD 113432, and HD 113422, the column densities obtained here are lower than the values $N_{\infty}(\mathrm{CH})$ of Paper I; this may reflect measurement uncertainties to a large extent. We use the averages obtained in the limit of $b \rightarrow \infty$ as final $\mathrm{CH}$ column densities toward the latter stars. Toward HD 114011, the saturation corrections of Paper I are probably too high as well. The column densities derived here and in Paper I in the limit of unsaturated lines agree, and we proceed to use the average obtained in the limit of $b(\mathrm{CH}) \rightarrow \infty$ as the final $\mathrm{CH}$ column density toward this star.

\section{Discussion}

Revised CH column densities obtained in Sect. 3.2 and final CN column densities obtained from the various methods discussed in Sect. 3.1 are summarised in Cols. 2 and 4, respectively, of Tables 7 and 8. For completeness, we add measured $\mathrm{CH}^{+}$and $\mathrm{C}_{2}$ column densities of Gredel (1997) and Gredel (1999) in Cols. 3 and 5, respectively, as well as the results for CMa OB1 and NGC 4755 from these papers. Heliocentric velocities for $\mathrm{CH}, \mathrm{CH}^{+}, \mathrm{CN}$, and $\mathrm{C}_{2}$ absorption are given in Cols. 6-9, respectively. In Col. 10 of these tables, we list the velocity of $\mathrm{CO}$ emission most likely associated with the molecular absorption. Tables 7 and 8 provide a coherent set of measurements of diatomic molecules toward the CMa OB1, NGC 2439, Vela OB1, NGC 4755, and Cen OB1 associations.

\subsection{Radial velocities of $\mathrm{CN}, \mathrm{C}_{2}, \mathrm{CH}$, and $\mathrm{CH}^{+}$}

In general, the measured radial velocities of $\mathrm{CN}$ agree well with those of $\mathrm{CH}, \mathrm{CH}^{+}, \mathrm{C}_{2}$, and $\mathrm{CO}$. Exceptions occur toward HD 75211 and $\mathrm{CPD}-44^{\circ} 3129$, where the $\mathrm{CN}$ radial velocity differs by some 6 and $4 \mathrm{~km} \mathrm{~s}^{-1}$, respectively, from that of the other molecules. The CN absorption lines toward HD 110639, HD 113432, and HD 114213 are resolved into two well separated components, and those toward HD 61827 are partially resolved. With the exception of $\mathrm{HD} 61827$, the $\mathrm{CH}^{+}$and $\mathrm{CH}$ absorption lines toward these stars are also resolved into two absorption components with similar heliocentric velocities. The $\mathrm{CH}$ and $\mathrm{CH}^{+}$lines in the spectrum of HD 61827 are relatively broad, with velocities that correspond to the average velocity of the two $\mathrm{CN}$ components. The $\mathrm{C}_{2}$ absorption lines toward HD 61827 are partly resolved into two components as well, and those toward HD 113422 and HD 113432 are resolved into two components. In all cases, the $\mathrm{C}_{2}$ radial velocities are similar to those of CN (Gredel 1999).

The $\mathrm{CH}$ radial velocities derived in Sect. 2.3 are generally in very good agreement with the measurements of 
Table 7. Final $\mathrm{CH}, \mathrm{CH}^{+}, \mathrm{CN}$, and $\mathrm{C}_{2}$ data for CMa OB1, NGC 2439, and Vela OB1.

\begin{tabular}{|c|c|c|c|c|c|c|c|c|c|}
\hline \multirow[t]{2}{*}{ star } & $N(\mathrm{CH})$ & $N\left(\mathrm{CH}^{+}\right)$ & $N(\mathrm{CN})$ & $N\left(\mathrm{C}_{2}\right)$ & $V_{\text {hel }}(\mathrm{CH})$ & $V_{\text {hel }}\left(\mathrm{CH}^{+}\right)$ & $V_{\text {hel }}(\mathrm{CN})$ & $V_{\text {hel }}\left(\mathrm{C}_{2}\right)$ & $V_{\text {hel }}(\mathrm{CO})$ \\
\hline & \multicolumn{4}{|c|}{$10^{13} \mathrm{~cm}^{-2}$} & \multicolumn{5}{|c|}{$\mathrm{km} \mathrm{s}^{-1}$} \\
\hline \multicolumn{10}{|l|}{ CMa OB1 } \\
\hline HD 55879 & $\leq 0.1$ & $0.35(0.10)$ & - & - & $24:$ & 23.8 & - & - & $24:$ \\
\hline HD 53975 & $<0.2$ & $0.3(0.2)$ & - & - & 33: & 33.4 & - & - & 33: \\
\hline HD 53755 & $0.3(0.2)$ & $0.9(0.2)$ & - & - & 26.9 & 29.8 & - & - & 35.0 \\
\hline HD 54662 & $0.8(0.1)$ & $1.1(0.1)$ & - & - & 35.8 & 35.5 & - & - & 32.6 \\
\hline HD 52382 & $1.1(0.1)$ & $2.4(0.2)$ & - & - & 36.0 & 37.2 & - & - & 37.7 \\
\hline \multicolumn{10}{|l|}{ NGC 2439} \\
\hline HD 63423 & $3.5(0.2)$ & $1.1(0.2)$ & $1.25_{-0.25}^{+0.45}$ & - & 40.5 & 39.4 & 41.1 & - & $40.6-41.4$ \\
\hline HD 62150 & $2.4(0.1)$ & $2.9(0.3)$ & $0.05(0.03)$ & - & 38.4 & 39.8 & 37.8 & - & 36.7 \\
\hline \multirow[t]{2}{*}{ HD 61827} & $2.6(0.1)$ & $1.3(0.1)^{a}$ & $0.36(0.07)$ & $2.8(0.5)$ & 36.3 & $38.3^{a}$ & 35.4 & 36.8 & 34.9 \\
\hline & $1.5(0.1)$ & $--^{a}$ & $0.36(0.07)$ & $3.8(0.5)$ & 41.7 & $-{ }^{a}$ & 41.4 & 43.4 & 41.0 \\
\hline HD 62844 & $5.0(0.5)$ & $3.2(0.2)$ & $0.31(0.06)$ & - & 39.1 & 39.7 & 38.1 & - & 39: \\
\hline $\mathrm{CPD}-32^{\circ} 1734$ & $9.0(2.0)$ & $6.0(0.5)$ & $1.0(0.2)$ & $10.5(1.0)$ & 37.8 & 37.8 & 38.7 & 37.8 & 38.3 \\
\hline $\mathrm{CPD}-33^{\circ} 1768$ & $12.0(3.0)$ & $4.4(0.5)$ & $0.95(0.10)$ & $11.0(1.5)$ & 38.6 & 43.3 & 40.6 & 41.5 & 39.7 \\
\hline HD $63804^{b}$ & $5.3(0.8)$ & $4.8(0.3)$ & $2.9_{-1.5}^{+5.0}$ & $10.0(4.0)$ & 37.7 & 37.2 & 37.6 & 39.5 & 37.0 \\
\hline \multicolumn{10}{|l|}{ Vela OB1 } \\
\hline HD 78344 & $6.0(0.5)$ & $3.5(0.4)$ & $2.67(0.50)$ & - & 19.5 & 20.9 & 20.2 & - & $19.6-21.4$ \\
\hline HD 74371 & $1.0(0.1)$ & $1.4(0.2)$ & $<0.05$ & - & 22.8 & 23.5 & $23:$ & - & $23:$ \\
\hline HD 75149 & $1.7(0.2)$ & $1.2(0.1)$ & $0.07(0.02)$ & $<0.5$ & 24.2 & 24.2 & 22.4 & $22:$ & $22:$ \\
\hline HD 74194 & $1.3(0.1)$ & $1.7(0.5)$ & $<0.05$ & - & 22.1 & 20.0 & $22:$ & - & $22:$ \\
\hline HD 75211 & $2.3(0.2)$ & $3.6(0.2)$ & $0.33(0.04)$ & - & 24.1 & 24.3 & 18.1 & - & $18.1-22.5$ \\
\hline HD 76556 & $4.0(0.5)$ & $1.5(0.2)$ & $1.18(0.20)$ & - & 19.9 & $4.0^{d}$ & 19.0 & - & 19.7 \\
\hline HD 75860 & $2.8(0.3)$ & $7.5(0.5)$ & $<0.05$ & $<0.5$ & 20.9 & 21.6 & $21:$ & 21: & $18.7-23.7$ \\
\hline $\mathrm{CPD}-44^{\circ} 3129$ & $5.3(0.5)$ & $6.3(1.5)$ & $0.78(0.10)$ & $3.3(1.5)$ & 21.3 & 22.8 & 18.0 & 21.9 & $18.7-21.2$ \\
\hline $\mathrm{CPD}-45^{\circ} 3218$ & $9.0(2.0)$ & $2.8(0.5)$ & $4.0(1.0)^{e}$ & - & 22.0 & 22.1 & 21.7 & - & $20.2-22.7$ \\
\hline $\mathrm{CPD}-46^{\circ} 3272$ & $6.0(1.0)$ & $3.2(0.5)$ & $1.4(0.1)^{e}$ & $6.0(1.5)$ & 21.3 & 22.1 & 22.0 & 21.4 & $19.7-23.3$ \\
\hline HD $73882^{b}$ & $3.7(0.5)$ & $2.4(0.3)$ & $3.8(0.4)$ & $3.5(1.0)$ & 21.3 & 21.5 & 21.3 & 21.8 & 22.6 \\
\hline
\end{tabular}

${ }^{a}$ Unresolved; 2 velocity components seen in $\mathrm{CH}, \mathrm{CN}, \mathrm{C}_{2}, \mathrm{CO}$.

${ }^{b}$ From Gredel et al. (1993), rescaled to $f_{\text {abs }}$ used here.

${ }^{c}$ Possibly affected by flexure from EMMI.

${ }^{d}$ Possible error in data reduction (see text).

e See text.

Gredel (1997). An exception occurs toward HD 76556, where our present value for $V_{\text {hel }}(\mathrm{CH})$ of $19.9 \mathrm{~km} \mathrm{~s}^{-1}$ is in clear contrast to the earlier value of $1.9 \mathrm{~km} \mathrm{~s}^{-1}$. Because the velocity inferred here agrees with that of $\mathrm{CN}$, $V_{\text {hel }}(\mathrm{CN})=19 \mathrm{~km} \mathrm{~s}^{-1}$, and because these velocities are similar to $\mathrm{CH}$ and $\mathrm{CN}$ radial velocities of other stars in Vela OB1, we suspect that the $\mathrm{CH}$ velocity given by Gredel (1997) toward HD 76556 is wrong. Possibly, the $\mathrm{CH}^{+}$velocity of $V_{\text {hel }}=+4 \mathrm{~km} \mathrm{~s}^{-1}$ toward that star is wrong as well, and we disregard the data toward HD 76556 in the following discussion.

Figure 6 contains a plot of the velocity differences $V(\mathrm{CN})-V(\mathrm{CH}), V(\mathrm{CN})-V\left(\mathrm{CH}^{+}\right), V(\mathrm{CN})-V\left(\mathrm{C}_{2}\right)$, and $V(\mathrm{CN})-V(\mathrm{CO})$ versus the velocity of $\mathrm{CN}$ obtained from the final heliocentric velocities given in Tables 7, 8 . In cases where ranges of velocities for $\mathrm{CO}$ are given, the central value is adopted as $\mathrm{CO}$ velocity. The scatter of some $1.5 \mathrm{~km} \mathrm{~s}^{-1}$ in the velocity differences reflect the uncertainties in the derived heliocentric velocities. Apart from two stars in Vela OB1, where the velocity of CN differs significantly from that of the other molecules, the velocity of $\mathrm{CH}^{+}$toward HD 113432 differs by $3.5-4 \mathrm{~km} \mathrm{~s}^{-1}$ from the velocity of $\mathrm{CN}$ and that of the other observed molecules. Toward all other sightlines studied here, the radial velocities of $\mathrm{CN}, \mathrm{CH}, \mathrm{C}_{2}, \mathrm{CO}$, and $\mathrm{CH}^{+}$agree within the errors. In cases where the $\mathrm{CO}$ velocities are spread over a range of velocities, the central $\mathrm{CO}$ velocities is used.

It has been debated in the past whether or not the velocities of the $\mathrm{CH}^{+}$lines show systematic shifts with respect to the velocities of lines from neutrals such as $\mathrm{CH}, \mathrm{CN}$, or $\mathrm{C}_{2}$. Velocity shifts up to a few $\mathrm{km} \mathrm{s}^{-1}$ between $\mathrm{CH}^{+}$and $\mathrm{CH}$ were suggested by models which 
Table 8. Final $\mathrm{CH}, \mathrm{CH}^{+}, \mathrm{CN}$, and $\mathrm{C}_{2}$ data for NGC 4755 and Cen OB1.

\begin{tabular}{|c|c|c|c|c|c|c|c|c|c|}
\hline \multirow[t]{2}{*}{ star } & $N(\mathrm{CH})$ & $N\left(\mathrm{CH}^{+}\right)$ & $N(\mathrm{CN})$ & $N\left(\mathrm{C}_{2}\right)$ & $V_{\text {hel }}(\mathrm{CH})$ & $V_{\text {hel }}\left(\mathrm{CH}^{+}\right)$ & $V_{\text {hel }}(\mathrm{CN})$ & $V_{\text {hel }}\left(\mathrm{C}_{2}\right)$ & $V_{\text {hel }}(\mathrm{CO})$ \\
\hline & \multicolumn{4}{|c|}{$10^{13} \mathrm{~cm}^{-2}$} & \multicolumn{5}{|c|}{$\mathrm{km} \mathrm{s}^{-1}$} \\
\hline \multicolumn{10}{|l|}{ NGC 4755} \\
\hline \multirow[t]{2}{*}{ HD 111973} & $0.3(0.1)$ & $0.4(0.1)$ & - & $<0.5$ & -10.6 & -13.3 & - & $-12:$ & \\
\hline & $0.5(0.1)$ & $0.9(0.1)$ & - & $<0.5$ & 7.8 & 8.6 & - & 8: & \\
\hline \multirow{2}{*}{ HD 111904} & $0.3(0.1)$ & $0.7(0.1)$ & - & - & -13.0 & -12.6 & & & \\
\hline & $0.4(0.1)$ & $0.5(0.1)$ & - & - & 8.2 & 8.6 & & & \\
\hline \multirow[t]{2}{*}{ HD 111934} & $0.4(0.1)$ & $0.6(0.1)$ & - & - & -10.6 & -11.9 & & & \\
\hline & $0.5(0.1)$ & $0.6(0.1)$ & - & - & 8.2 & 8.6 & & & \\
\hline \multirow[t]{2}{*}{ HD 111990} & $0.4(0.1)$ & $0.9(0.1)$ & - & - & -10.6 & -14.0 & & & \\
\hline & $0.7(0.1)$ & $0.6(0.1)$ & - & - & 8.2 & 7.9 & & & \\
\hline \multirow[t]{2}{*}{$\mathrm{CPD}-59^{\circ} 4551$} & $0.7(0.2)$ & $1.8(0.1)$ & - & - & -11.2 & -11.2 & & & \\
\hline & $0.7(0.2)$ & $0.9(0.1)$ & - & - & 8.2 & 7.2 & & & \\
\hline \multicolumn{10}{|l|}{ Cen OB1 } \\
\hline \multirow[t]{2}{*}{ HD 114886} & $0.4(0.1)$ & $0.6(0.1)$ & - & - & -21.8 & -20.4 & - & - & - \\
\hline & $0.5(0.2)$ & $1.4(0.1)$ & - & - & 4.1 & 2.3 & - & - & - \\
\hline \multirow[t]{2}{*}{ HD 115704} & $1.3(0.1)$ & $2.3(0.2)$ & - & - & -28.0 & -25.1 & - & - & -30.5 \\
\hline & $0.5(0.1)$ & $0.5(0.2)$ & - & - & 1.2 & 2.6 & - & - & $2:$ \\
\hline \multirow[t]{2}{*}{ HD 112366} & $<0.4$ & $1.3(0.2)$ & - & - & $-14:$ & -13.9 & - & - & $-10.8--18.4$ \\
\hline & $3.8(0.5)$ & $0.8(0.1)$ & $1.90(0.20)$ & - & 5.3 & 4.3 & 5.4 & - & 6.2 \\
\hline \multirow[t]{2}{*}{ HD 115363} & $1.1(0.1)$ & $0.9(0.2)$ & $<0.05$ & - & -22.5 & -20.4 & $-22:$ & - & -22.0 \\
\hline & $2.5(0.2)$ & $0.9(0.2)$ & $0.28(0.02)$ & - & 4.2 & 4.4 & 3.8 & - & 4.3 \\
\hline \multirow[t]{2}{*}{ HD 110639} & $2.0(0.2)$ & $1.2(0.1)$ & $0.16(0.05)$ & $<0.5$ & -20.3 & -19.6 & -20.6 & $-20.5:$ & -20.5 \\
\hline & $1.4(0.1)$ & $2.4(0.2)$ & $0.57(0.05)$ & $<0.5$ & 3.9 & 4.4 & 2.9 & 3.5: & 3.5 \\
\hline \multirow[t]{2}{*}{ HD 114011} & $4.7(0.4)$ & $2.0(0.4)$ & $3.79(0.40)$ & $7.5(1.0)$ & -25.9 & -23.9 & -24.9 & -26.4 & -24.7 \\
\hline & $<0.5$ & $0.6(0.1)$ & - & $<0.5$ & 4: & 0.1 & - & 4: & 4: \\
\hline \multirow[t]{2}{*}{ HD 113432} & $1.1(0.2)$ & $2.2(0.4)$ & - & $1.2:$ & -17.6 & -19.0 & - & $-15.2:$ & -18.3 \\
\hline & $3.7(0.2)$ & $1.4(0.4)$ & $1.88(0.30)$ & 1.6: & 4.1 & 0.9 & 4.7 & 4.6: & $2.4-3.6$ \\
\hline \multirow[t]{2}{*}{ HD 113422} & $2.6(0.3)$ & $4.0(0.3)$ & $0.32(0.05)$ & $3.0(1.0)$ & -18.2 & -18.2 & -18.5 & -17.8 & -18.8 \\
\hline & $1.6(0.2)$ & $1.6(0.2)$ & $0.44(0.10)$ & $<0.8$ & 3.4 & 3.7 & 3.2 & 2.7: & 3.4 \\
\hline \multirow[t]{2}{*}{ HD 112272} & $2.8(0.2)$ & $2.5(0.3)$ & - & - & -8.2 & -7.1 & - & - & -10.2 \\
\hline & $2.8(0.2)$ & $1.3(0.2)$ & $0.18(0.04)$ & - & 4.0 & 6.1 & 5.0 & - & 5.3 \\
\hline \multirow[t]{2}{*}{ HD 114213} & $4.1(0.3)$ & $1.3(0.4)$ & $4.68(1.00)$ & $3.5(0.5)$ & -20.0 & -20.4 & -20.0 & -20.8 & -19.6 \\
\hline & $1.8(0.8)$ & $<0.5$ & $0.28(0.08)$ & $<0.5$ & 2.4 & $2.4:$ & 3.2 & 3.6: & 3.6 \\
\hline
\end{tabular}

attempted to explain $\mathrm{CH}^{+}$formation in magnetic hydrodynamic shocks (Pineau des Forêts et al. 1986). Flower \& Pineau des Forêts (1998) have eased the earlier constraints and purported a way in which the $\mathrm{CH}^{+}$velocity can be brought in agreement with that of $\mathrm{CH}$. The reaction rates adopted by Flower \& Pineau des Forêts (1998) imply that $\mathrm{CH}$ is efficiently removed in the cool gas, and that significant fractions of interstellar $\mathrm{CH}$ form in the hot post-shock gas as well (c.f. discussion in Gredel 1999), at elevated temperatures of many $100 \mathrm{~K}$; this material is rich in $\mathrm{CH}^{+}$. However, the empirically established, tight correlation between the $\mathrm{CH}$ and $\mathrm{C}_{2}$ column densities (Federman et al. 1994; Gredel 1999), together with the very low gas-kinetic temperatures of a few ten $\mathrm{K}$ derived from $\mathrm{C}_{2}$ excitation (Gredel 1999), is difficult to explain in terms of a significant formation of $\mathrm{CH}$ in hot material. The ultra-high resolution observations of Lambert et al. (1990) and others show that the $\mathrm{CH}$ line profile may be described in terms of a $\mathrm{CH}^{+}$-like component, with rather large width, and a CN-like component, with a narrow width. Gredel (1999) estimated an upper limit of some $20 \%$ of $\mathrm{CH}$ in $\mathrm{CH}^{+}$-like gas toward the southern associations. In Sect. 4.3, we introduce an ad hoc component of some 10-50 criss-crossing MHD shocks where the amount of $\mathrm{CH}^{+}$-like $\mathrm{CH}$ is restricted to observe this limit.

\subsection{Chemistry of $\mathrm{CN}$ in quiescent gas}

We extracted gas densities, $n=2 n\left(\mathrm{H}_{2}\right)+n(\mathrm{H})$, from the observational results on $\mathrm{CN}$ presented in Tables 7 and 8 in conjunction with those on $\mathrm{CH}$ and $\mathrm{C}_{2}$ (when the latter were available) with an updated version of the chemical model described by Federman et al. (1994). The updates, which were needed to keep reaction rates the same as before when using current values for the abundance of $\mathrm{C}^{+}$(Sofia et al. 1997), are discussed in detail elsewhere (Knauth et al. 2001; Pan et al. 2001). The synthesis of $\mathrm{CN}$ along the sight lines studied here arises from $\mathrm{N}$ reacting with $\mathrm{CH}$ and $\mathrm{C}_{2}$, while the production of $\mathrm{C}_{2}$ is initiated by the reaction $\mathrm{C}^{+}+\mathrm{CH} \rightarrow \mathrm{C}_{2}^{+}+\mathrm{H}$. The main destruction pathways for $\mathrm{CN}$ and $\mathrm{C}_{2}$ are photodissociation 


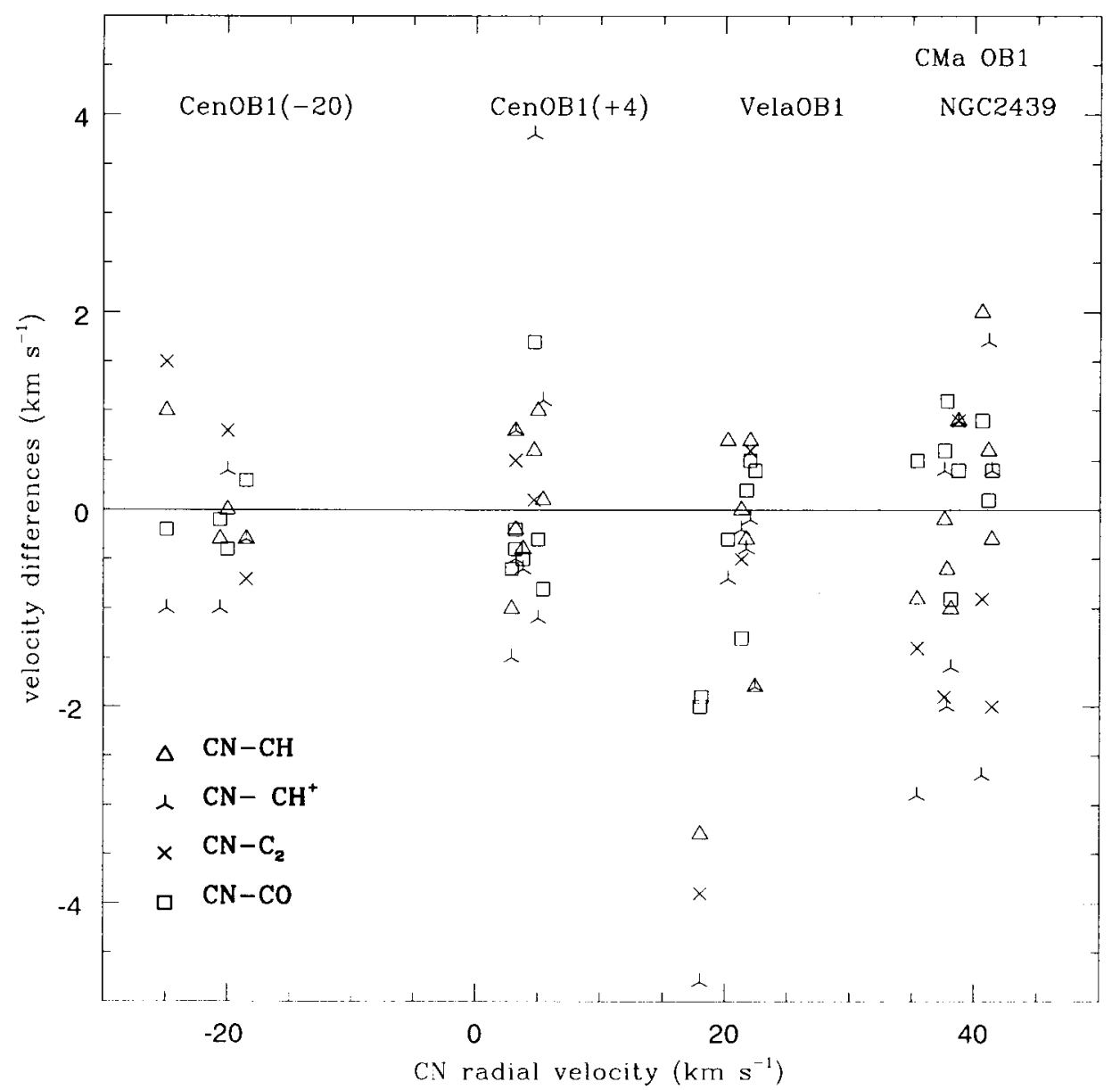

Fig. 6. Velocity differences $V(\mathrm{CN})-V(\mathrm{CH}), V(\mathrm{CN})-V\left(\mathrm{CH}^{+}\right), V(\mathrm{CN})-V\left(\mathrm{C}_{2}\right)$, and $V(\mathrm{CN})-V(\mathrm{CO})$ plotted versus the velocity of $\mathrm{CN}$.

and reactions with atomic $\mathrm{O}$ and $\mathrm{N}$, the latter being relevant for $\mathrm{C}_{2}$. The parameter $\alpha$, which is used to estimate the conversion of $\mathrm{C}^{+}$into $\mathrm{CO}$ as the extinction within a cloud increases, is especially important for these reddened sight lines; it is given by $\left[1+14\left(\tau_{\mathrm{UV}}-2\right) / 5\right]^{-1}$. The determination of $N\left(\mathrm{C}_{2}\right)$ is based on the observed amount of $\mathrm{CH}$ and that of $N(\mathrm{CN})$ on the observed amounts of $\mathrm{CH}$ and $\mathrm{C}_{2}$. For sight lines without measurements on $\mathrm{C}_{2}$, the predicted amount of $\mathrm{C}_{2}$ is used in the derivation for $N(\mathrm{CN})$.

The calculations proceeded under the following conditions. The kinetic temperature for all clouds was set to $50 \mathrm{~K}$, but we note that the results are not very sensitive to its value. The fractional abundances for $\mathrm{C}^{+}, \mathrm{N}$, and $\mathrm{O}$ in unshielded regions were $1.4 \times 10^{-4}$ (Sofia et al. 1997), $7.5 \times 10^{-5}$ (Meyer et al. 1997), and $3.2 \times 10^{-4}$ (Meyer et al. 1998), respectively. The value for $\tau_{U V}$ was set equal to $2 A_{V}$ (Federman et al. 1994), but for directions with 2 absorption components separated by at least $20 \mathrm{~km} \mathrm{~s}^{-1}$, each component was assumed to have half the total extinction for the line of sight. Density estimates were obtained for models with a UV flux equal to the average interstellar value $\left(I_{\mathrm{UV}}=1\right)$ and with a flux that is a factor of 5 stronger. Since a factor-of- 5 increase in the $\mathrm{UV}$ flux is expected to increase the abundance of $\mathrm{C}^{+}$(at the expense of $\mathrm{CO}$ ) for moderately reddened directions, the model had to accommodate this possibility. For sight lines with $\tau_{\text {UV }}$ greater than 4 , the factor-of- 5 increase in flux was accomplished by decreasing $\tau_{\mathrm{UV}}$ by 1.61 so that $\alpha$ remained closer to 1 . Finally, since the chemical scheme pertains to diffuse molecular clouds, models with densities greater than $1600 \mathrm{~cm}^{-3}$ were not considered.

The results of our analysis appear in Tables 9 and 10. Densities typical of diffuse molecular gas (100 to $1400 \mathrm{~cm}^{-3}$ ) are obtained for most of the sightlines in our study, usually for both choices of enhancement factor in the UV flux. Lower limits of $1600 \mathrm{~cm}^{-3}$ are inferred toward $\mathrm{CPD}-32^{\circ} 1734$ and $\mathrm{CPD}-33^{\circ} 1768$, indicating that $\mathrm{CN}$ is mainly produced under dark cloud conditions. The same seems to apply for the gas toward HD 114011, where the results based on a density of $1600 \mathrm{~cm}^{-3}$ are lower than the observed $\mathrm{CN}$ and $\mathrm{C}_{2}$ columns, but within their uncertainties. Dark cloud chemistry also appears to be operating when the model can reproduce the $\mathrm{C}_{2}$ column, but falls short for CN, such as toward HD 73882, HD 110639 at $+3 \mathrm{kms}^{-1}$, HD 113432, HD 113422 at $+3 \mathrm{~km} \mathrm{~s}^{-1}$, and HD 114213 at $-20 \mathrm{~km} \mathrm{~s}^{-1}$. For sightlines with upper limits on the column of CN [HD 74371, HD 74194, HD 75860, and HD 115363(-22)], the densities are found to be less than about $200 \mathrm{~cm}^{-3}$ (for $I_{\mathrm{UV}}$ of 1 ). Since $\mathrm{C}_{2}$ absorption is 
Table 9. Chemical analysis for gas toward NGC 2439 and Vel OB1.

\begin{tabular}{|c|c|c|c|c|c|c|c|c|c|}
\hline \multirow[t]{2}{*}{ star } & \multirow[t]{2}{*}{$\tau_{\mathrm{UV}}$} & \multicolumn{2}{|c|}{$n\left(\mathrm{~cm}^{-3}\right)$} & \multicolumn{3}{|c|}{$N\left(\mathrm{C}_{2}\right)\left(10^{13} \mathrm{~cm}^{-2}\right)$} & \multicolumn{3}{|c|}{$N(\mathrm{CN})\left(10^{13} \mathrm{~cm}^{-2}\right)$} \\
\hline & & $I_{\mathrm{UV}}=1$ & $I_{\mathrm{UV}}=5$ & Observed & $I_{\mathrm{UV}}=1$ & $I_{\mathrm{UV}}=5$ & Observed & $I_{\mathrm{UV}}=1$ & $I_{\mathrm{UV}}=5$ \\
\hline \multicolumn{10}{|l|}{ NGC 2439} \\
\hline HD 63423 & 3.06 & 1325 & $>1600$ & $\ldots$ & 5.2 & $\ldots$ & $1.25_{-0.25}^{+0.45}$ & 1.25 & ... \\
\hline HD 62150 & 3.54 & 50 & 250 & $\ldots$ & 0.3 & 0.3 & $0.05(0.03)$ & 0.05 & 0.05 \\
\hline HD $61827(+35)$ & 4.20 & 200 & 600 & $2.8(0.5)$ & 1.3 & 2.4 & $0.36(0.07)$ & 0.42 & 0.40 \\
\hline HD $61827(+41)$ & 4.20 & 250 & 925 & $3.8(0.5)$ & 0.9 & 2.0 & $0.36(0.07)$ & 0.42 & 0.43 \\
\hline HD 62844 & 5.34 & 30 & 125 & $\ldots$ & 1.0 & 1.5 & $0.31(0.06)$ & 0.27 & 0.29 \\
\hline $\mathrm{CPD}-32^{\circ} 1734$ & 7.32 & $\ldots$ & $>1600$ & $10.5(0.5)$ & $\ldots$ & $\ldots$ & $1.0(0.2)$ & . & $\ldots$ \\
\hline $\mathrm{CPD}-33^{\circ} 1768$ & 8.76 & $\ldots$ & $>1600$ & $11.0(1.5)$ & . & . . & $0.95(0.10)$ & . & $\ldots$ \\
\hline HD 63804 & 7.14 & 175 & 750 & $10.0(4.0)$ & 3.9 & 5.4 & $2.9_{-1.5}^{+5.0}$ & 3.4 & 3.4 \\
\hline \multicolumn{10}{|l|}{ Vela OB1 } \\
\hline HD 78344 & 8.40 & 150 & 275 & $\ldots$ & 4.0 & 4.8 & $2.67(0.50)$ & 2.66 & 2.67 \\
\hline HD 74371 & 1.86 & $<225$ & $<1075$ & $\ldots$ & $<0.5$ & $<0.5$ & $<0.05$ & $<0.05$ & $<0.05$ \\
\hline HD 75149 & 2.28 & 175 & 825 & $<0.5$ & 0.6 & 0.6 & $0.07(0.02)$ & 0.07 & 0.06 \\
\hline HD 74194 & 3.24 & $<125$ & $<575$ & $\ldots$ & $<0.3$ & $<0.3$ & $<0.05$ & $<0.05$ & $<0.05$ \\
\hline HD 75211 & 4.32 & 200 & 575 & $\ldots$ & 1.2 & 2.0 & $0.33(0.04)$ & 0.33 & 0.34 \\
\hline HD 76556 & 4.38 & 475 & 1175 & $\ldots$ & 3.6 & 5.8 & $1.18(0.20)$ & 1.16 & 1.19 \\
\hline HD 75860 & 5.58 & $<20$ & $<30$ & $<0.5$ & $<0.4$ & $<0.3$ & $<0.05$ & $<0.13$ & $<0.05$ \\
\hline $\mathrm{CPD}-44^{\circ} 3129$ & 6.20 & 40 & 150 & $3.3(1.5)$ & 2.0 & 2.5 & $0.78(0.10)$ & 0.83 & 0.75 \\
\hline CPD $-45^{\circ} 3218$ & 6.36 & 350 & 825 & $\ldots$ & 7.7 & 10.0 & $4.0(1.0)$ & 4.0 & 4.0 \\
\hline $\mathrm{CPD}-46^{\circ} 3272$ & 6.36 & 70 & 275 & $6.0(1.5)$ & 3.2 & 4.3 & $1.4(0.1)$ & 1.7 & 1.6 \\
\hline HD 73882 & 4.32 & 875 & $\ldots$ & $3.5(1.0)$ & 4.3 & $\ldots$ & $3.8(0.4)$ & 1.4 & $\ldots$ \\
\hline
\end{tabular}

Table 10. Chemical analysis for gas toward Cen OB1.

\begin{tabular}{|c|c|c|c|c|c|c|c|c|c|}
\hline \multirow[t]{2}{*}{ star } & \multirow[t]{2}{*}{$\tau_{\mathrm{UV}}$} & \multicolumn{2}{|c|}{$n\left(\mathrm{~cm}^{-3}\right)$} & \multicolumn{3}{|c|}{$N\left(\mathrm{C}_{2}\right)\left(10^{13} \mathrm{~cm}^{-2}\right)$} & \multicolumn{3}{|c|}{$N(\mathrm{CN})\left(10^{13} \mathrm{~cm}^{-2}\right)$} \\
\hline & & $I_{\mathrm{UV}}=1$ & $I_{\mathrm{UV}}=5$ & Observed & $I_{\mathrm{UV}}=1$ & $I_{\mathrm{UV}}=5$ & Observed & $I_{\mathrm{UV}}=1$ & $I_{\mathrm{UV}}=5$ \\
\hline \multicolumn{10}{|l|}{ Cen OB1 } \\
\hline HD 112366 & 4.50 & 1250 & $>1600$ & $\ldots$ & 4.8 & $\ldots$ & $1.90(0.20)$ & 1.91 & \\
\hline HD $115363(-22)$ & $2.58^{a}$ & $<200$ & $<975$ & $\ldots$ & $<0.4$ & $<0.4$ & $<0.05$ & $<0.05$ & $<0.05$ \\
\hline HD $115363(+4)$ & $2.58^{a}$ & 500 & $>1600$ & $\ldots$ & 2.0 & & $0.28(0.02)$ & 0.3 & \\
\hline HD $110639(-21)$ & $2.73^{a}$ & 175 & 850 & $<0.5$ & 0.6 & 0.6 & $0.16(0.05)$ & 0.09 & 0.08 \\
\hline HD $110639(+3)$ & $2.73^{a}$ & 225 & 1150 & $<0.5$ & 0.5 & 0.6 & $0.57(0.05)$ & 0.08 & 0.08 \\
\hline HD 114011 & 5.88 & $>1600$ & $>1600$ & $7.5(1.0)$ & $\ldots$ & 6.5 & $3.79(0.40)$ & $\ldots$ & 2.67 \\
\hline HD 113432 & 6.12 & 80 & 175 & 1.6: & 2.0 & 1.9 & $1.88(0.30)$ & 0.77 & 0.51 \\
\hline HD $113422(-19)$ & $3.24^{a}$ & 375 & $>1600$ & $3.0(1.0)$ & 1.7 & $\ldots$ & $0.32(0.03)$ & 0.38 & . \\
\hline HD $113422(+3)$ & $3.24^{a}$ & 275 & 1400 & $<0.8$ & 0.8 & 0.8 & $0.44(0.10)$ & 0.15 & 0.15 \\
\hline HD $112272(+5)$ & 6.54 & $\ldots$ & 50 & $\ldots$ & $\ldots$ & 0.7 & $0.18(0.04)$ & $\ldots$ & 0.18 \\
\hline HD $114213(-20)$ & $3.42^{a}$ & 675 & $>1600$ & $3.5(0.5)$ & 4.0 & $\ldots$ & $4.68(1.00)$ & 0.93 & $\ldots$ \\
\hline HD $114213(+3)$ & $3.42^{a}$ & 175 & 825 & $<0.5$ & 0.6 & 0.6 & $0.28(0.08)$ & 0.11 & 0.11 \\
\hline
\end{tabular}

${ }^{a}$ Each component has $1 / 2$ the extinction along the line of sight.

not observed for any of these sightlines either, the amount of $\mathrm{CH}$ is probably controlled by the synthesis of $\mathrm{CH}^{+}$in relatively low density gas. Ellipses in Tables 9 and 10 are given where the modeling results cannot be determined under the circumstances. One concern remains when analyzing these data: how much of the extinction in these reddened sight lines comes from the molecule-rich gas? Unfortunately, no measures of H I content are available, and so we had to assume that the extinction arose from one cloud (unless there were distinct velocity components separated by more than $20 \mathrm{~km} \mathrm{~s}^{-1}$ ). Use of lower extinctions per cloud would result in higher inferred densities because photodissociation would play a more important role. The inferred densities are in excellent agreement with densities inferred from $\mathrm{C}_{2}$ excitation (Gredel et al. 1993; Gredel 1999).

\section{3. $\mathrm{CH}^{+}$production in $\mathrm{MHD}$ shocks}

The formation of $\mathrm{CH}^{+}$in translucent clouds has been studied by many authors involving different scenarios with mixed success: MHD shocks (Draine \& Katz 1986; Pineau des Forêts et al. 1986), turbulent boundary layers (Duley et al. 1992), non-maxwellian velocity distributions (Spaans 1995) or interstellar turbulence 
(Falgarone et al. 1995; Joulain et al. 1998). All these processes have been reviewed in the context of $\mathrm{CH}^{+}$ formation by Gredel (1997). In practice, these models have difficulties in reproducing, all together, the observational constraints: (1) the correlation between the column density $N\left(\mathrm{CH}^{+}\right)$and that of rotationally excited $\mathrm{H}_{2}$, (2) the trend of $N\left(\mathrm{CH}^{+}\right)$with visual extinction of the background star and with $\mathrm{CH}$ column density, and (3) the absence of a significant $\mathrm{CH}-\mathrm{CH}^{+}$velocity shift. Furthermore, the observations show that this velocity shift does not increase with $\mathrm{CH}^{+}$column density. The purpose of the present approach is to provide a model consistent with these different constraints.

Flower \& Pineau des Forêts (1998) calculated CH and $\mathrm{CH}^{+}$line profiles in $\mathrm{C}$-type shocks and showed that the predicted velocity shift is much smaller than had been assumed previously. $\mathrm{CH}$ is formed via dissociative recombination, $\mathrm{CH}_{3}^{+}\left(\mathrm{e}, \mathrm{H}_{2}\right) \mathrm{CH}$, at the ion flow speed but is rapidly destroyed through the reaction $\mathrm{CH}\left(\mathrm{H}, \mathrm{H}_{2}\right) \mathrm{C}$ before being thermalized by collisions with the neutrals, leading to a velocity of $\mathrm{CH}$ close to the ion $\left(\mathrm{CH}^{+}\right)$speed. A single $\mathrm{C}$-shock along a given line of sight could then explain most of the observed $\mathrm{CH}^{+}$column densities. The scaling relation between observed $\mathrm{CH}^{+}$column density and visual extinction is approximately $N\left(\mathrm{CH}^{+}\right)=10^{13} \times A_{\mathrm{V}} \mathrm{cm}^{-2}$; it could be explained by one $\mathrm{C}$-shock with a velocity of $12 \mathrm{~km} \mathrm{~s}^{-1}$ leading to a predicted velocity shift between $\mathrm{CH}^{+}$and $\mathrm{CH}$ of less than $2 \mathrm{~km} \mathrm{~s}^{-1}$. However, as proposed by Gredel (1997), it is more likely that several shock waves will be present along the line of sight, with lower velocities, and that the number correlates with the optical depth of the clouds.

We considered a model of MHD shocks that was described by Flower \& Pineau des Forêts (1998). We extended the chemical network to 97 chemical species containing $\mathrm{H}, \mathrm{He}, \mathrm{C}, \mathrm{N}, \mathrm{O}, \mathrm{S}$ and a representative metal linked by approximately 800 chemical reactions. The initial elemental abundances were taken from Meyer (1997). To illustrate the dissipation of kinetic energy through a number of low velocity shocks along the lines of sight, we used only two C-shock models with velocities of 8 and $9 \mathrm{~km} \mathrm{~s}^{-1}$ propagating into a preshock gas of density $n=20 \mathrm{~cm}^{-3}$ in which the transverse magnetic induction is assumed to be $5 \mu \mathrm{G}$.

The preshock gas is supposed to be at steady state, at a temperature of $80 \mathrm{~K}$, and illuminated by the standard local interstellar radiation field. To calculate the preshock abundances of the species, we used an updated version of the Photon Dominated Region (PDR) model of Le Bourlot et al. (1993). For a visual extinction $A_{\mathrm{V}}=$ 0.1 , the model yields an $\mathrm{H}_{2} / \mathrm{H}$ ratio in the preshock gas of approximately $0.15 . \mathrm{H}_{2}$ and $\mathrm{CO}$ photodissociation rates from the PDR model are expressed as functions of $A_{\mathrm{V}}$ and then are introduced into the shock model. A more detailed description of the shock model and of the main chemical reactions involved can be found in Flower \& Pineau des Forêts (1998).

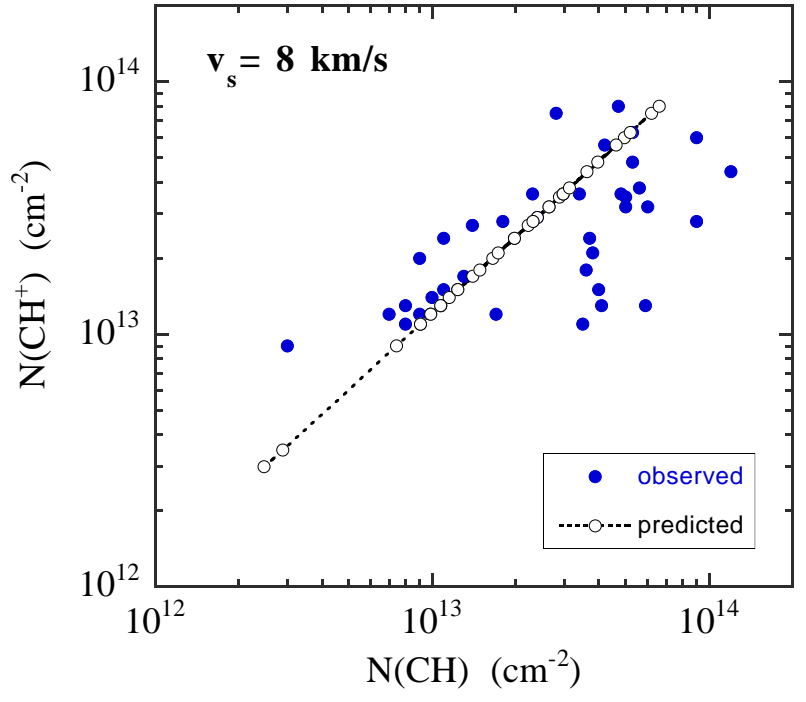

Fig. 7. $\mathrm{CH}^{+}$versus $\mathrm{CH}$ column densities in shocks of $V_{\mathrm{s}}=$ $8 \mathrm{~km} \mathrm{~s}^{-1}$.

We calculated the number of shocks necessary to reproduce the observed $\mathrm{CH}^{+}$column density toward the different lines of sight. Tables 11 and 12 contain the resulting $\mathrm{CH}$ and $\mathrm{CH}^{+}$column densities. Columns 1 and 2 contain the star and the total visual extinction along the line of sight. Columns 3 and 4 give the fraction of gas (in units of $A_{\mathrm{V}}$ ) in the MHD component. The fraction of gas in the shocked gas varies from sightline to sightline and with the velocities of the shocks, but the total amount of gas in the hot component is small in all cases. Columns 5 and 6 give the number of shocks per sightline, $N_{\mathrm{s}}$, for shock velocities $V_{\mathrm{s}}$ of 8 and $9 \mathrm{~km} \mathrm{~s}^{-1}$, respectively.

The observed $\mathrm{CH}$ and $\mathrm{CH}^{+}$column densities are given in Cols. 7 and 10. Columns 8 and 9 contain the column density of $\mathrm{CH}$ produced in shocks of 8 and $9 \mathrm{~km} \mathrm{~s}^{-1}$, respectively, and Cols. 11 and 12 that of $\mathrm{CH}^{+}$. The number of shocks toward each line of sight is chosen to reproduce the observed $\mathrm{CH}^{+}$column densities. The amount of $\mathrm{CH}^{+}$-like $\mathrm{CH}$, that is, the amount of $\mathrm{CH}$ produced in the MHD shocks, is very large for low shock velocities $\left(V_{\mathrm{s}} \leq 7 \mathrm{~km} \mathrm{~s}^{-1}\right)$. This is illustrated in Figs. 7 and 8 , where the predictions of the models, varying the number of shocks, are compared to the observations. For lower shock velocities, there are a greater number of shocks along the line of sight, with larger $\mathrm{CH}$ column densities (i.e. the $N(\mathrm{CH}) / N\left(\mathrm{CH}^{+}\right)$ratio). The consequence is that for lower velocities (i.e. 6 or $7 \mathrm{~km} \mathrm{~s}^{-1}$ ) too much $\mathrm{CH}$ is produced in the MHD component, unless the magnetic field is reduced. For shock speeds above $8 \mathrm{~km} \mathrm{~s}^{-1}$, a fraction of $10-30 \%$ of $\mathrm{CH}^{+}$-like $\mathrm{CH}$ is obtained in general.

As is demonstrated in Figs. 9 and 10, $N_{\mathrm{s}}$ is well distributed around 20 shocks of velocity $8 \mathrm{~km} \mathrm{~s}^{-1}$ per $A_{\mathrm{V}}$ and 5 shocks of velocity $9 \mathrm{~km} \mathrm{~s}^{-1}$ per $A_{\mathrm{V}}$ respectively. We emphasise that this approach is only an illustration and a mixture of shocks with various velocities (and directions) may be present along the different lines of sight. It is nevertheless obvious that the number $N_{\mathrm{s}}$ varies from one line 


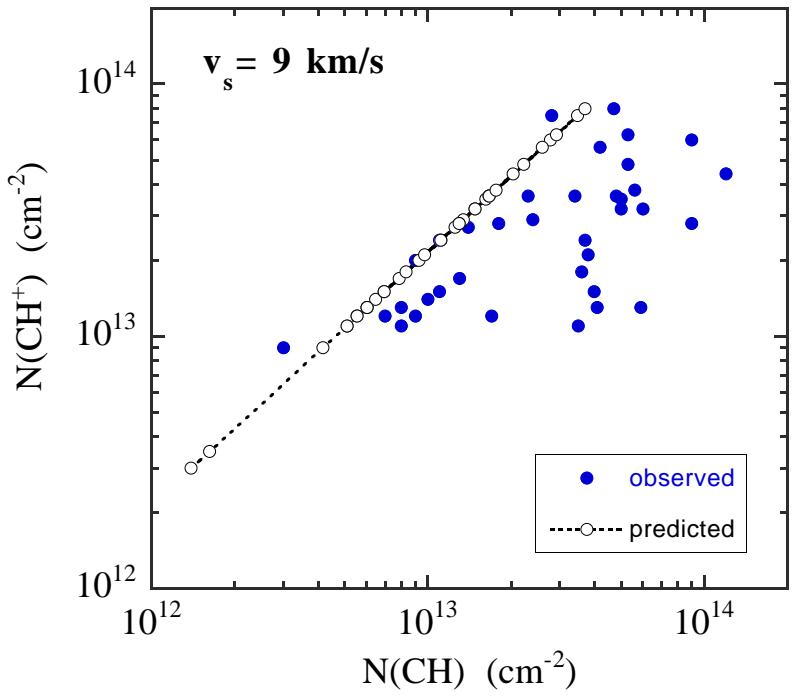

Fig. 8. $\mathrm{CH}^{+}$versus $\mathrm{CH}$ column densities in shocks of $V_{\mathrm{s}}=$ $9 \mathrm{kms}^{-1}$.

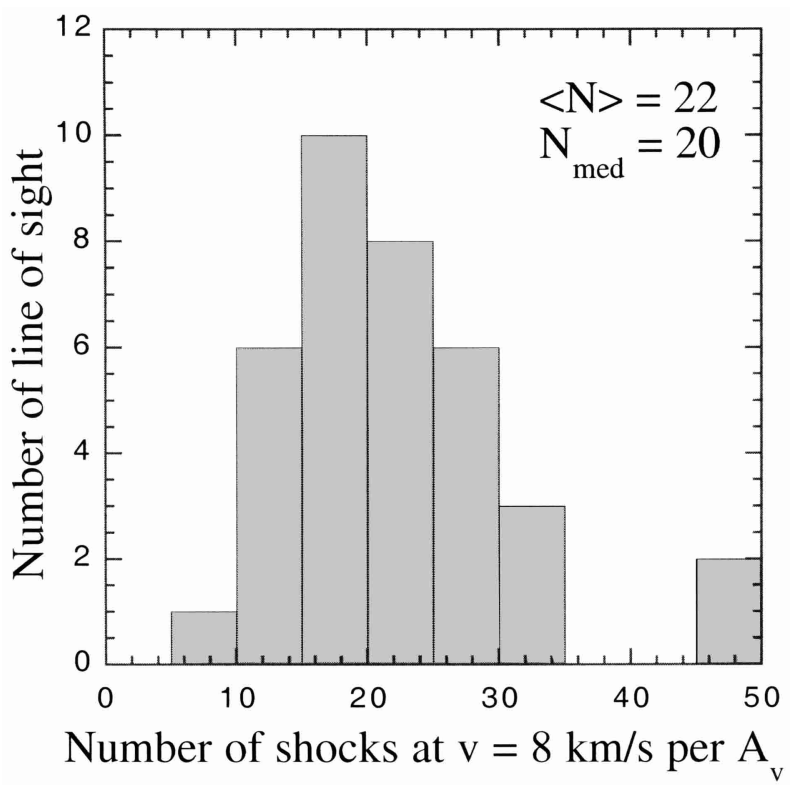

Fig. 9. Number of shocks per sightline for $V_{\mathrm{s}}=8 \mathrm{~km} \mathrm{~s}^{-1}$ required in model to reproduce observed column densities of $\mathrm{CH}^{+}$.

of sight to another and is not, then, an "interstellar constant".

We also give in Tables 11 and 12 the predicted columns of $\mathrm{CO}$ and $\mathrm{OH}$ in Cols. 13-16, again for shock speeds of 8 and $9 \mathrm{kms}^{-1}$. Observational confirmation of these predicted column densities would be an important test of the present model. The amount of $\mathrm{CN}$ and $\mathrm{C}_{2}$ produced in the shocks is very low and stays below column densities of $10^{12} \mathrm{~cm}^{-2}$ in all cases.

As an illustration, the computed absorption line profiles for $\mathrm{CH}$ and $\mathrm{CH}^{+}$in a single shock of velocity $8 \mathrm{~km} \mathrm{~s}^{-1}$ (using a microturbulent velocity $b=2 \mathrm{~km} \mathrm{~s}^{-1}$ ) are presented in Fig. 11 where velocities are expressed in the

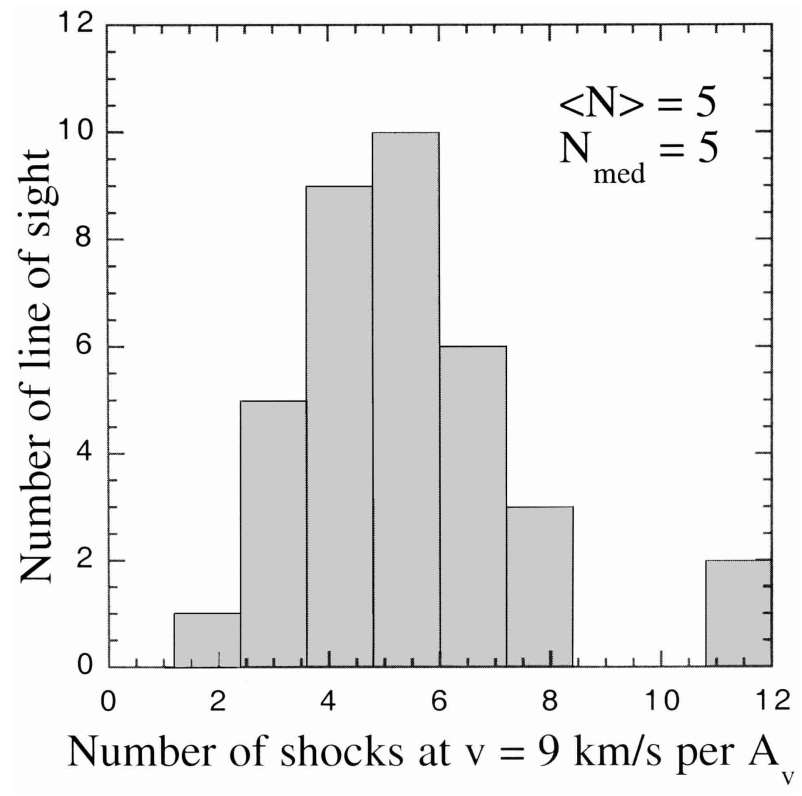

Fig. 10. Number of shocks per sightline for $V_{\mathrm{s}}=9 \mathrm{~km} \mathrm{~s}^{-1}$ required in model to reproduce observed column densities of $\mathrm{CH}^{+}$.

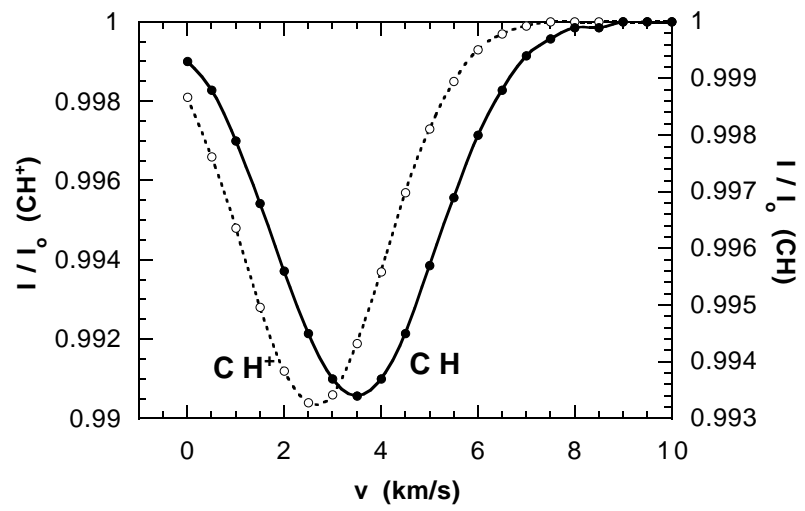

Fig. 11. $\mathrm{CH}$ and $\mathrm{CH}^{+}$absorption line profiles in an $8 \mathrm{~km} \mathrm{~s}^{-1}$ MHD shock.

shock frame. It is clear that even for a shock propagating along the line of sight, the velocity shift between $\mathrm{CH}$ and $\mathrm{CH}^{+}$is smaller than $1 \mathrm{~km} \mathrm{~s}^{-1}$. Furthermore, for shocks propagating in random directions along the line of sight, the effect of projection reduces the kinematic broadening (and hence the width of the $\mathrm{CH}$ line relative to that of $\mathrm{CH}^{+}$), as well as the shift between $\mathrm{CH}$ and $\mathrm{CH}^{+}$(Flower \& Pineau des Forêts 1998).

When some of the $\mathrm{CH}$ arises from MHD shocks, less of the observed $\mathrm{CH}$ column comes from the quiescent gas. We investigated the effect of reduced $\mathrm{CH}$ column by subtracting the amount of $\mathrm{CH}^{+}$-like $\mathrm{CH}$ produced by $9 \mathrm{~km} \mathrm{~s}^{-1}$ shocks (cf. Col. 9 of Tables 11 and 12) from the total observed $\mathrm{CH}$ column density and iterating the analysis of Sect. 4.2. Generally, when $\mathrm{C}_{2}$ and $\mathrm{CN}$ photodissociation dominate, the inferred densities given in Tables 9 and 10 scale inversely with $N(\mathrm{CH})$; a larger $n$ is needed to compensate for a reduced $N(\mathrm{CH})$. This situation applies to 
Table 11. Modeled $\mathrm{CH}, \mathrm{CH}^{+}, \mathrm{CO}$, and $\mathrm{OH}$ column densities in the hot MHD component toward CMa OB1, NGC 2439, and Vel OB1.

\begin{tabular}{|c|c|c|c|c|c|c|c|c|c|c|c|c|c|c|c|}
\hline \multirow{3}{*}{$\begin{array}{l}\text { star } \\
\text { HD/CPD }\end{array}$} & \multirow{2}{*}{\multicolumn{3}{|c|}{$A_{\mathrm{V}}(\mathrm{mag})$}} & \multirow{2}{*}{\multicolumn{2}{|c|}{$N_{\mathrm{s}}$}} & \multirow{2}{*}{\multicolumn{3}{|c|}{$\frac{N(\mathrm{CH})}{0^{13} \mathrm{~cm}^{-8}}$}} & \multirow{2}{*}{\multicolumn{3}{|c|}{$\frac{N\left(\mathrm{CH}^{+}\right)}{10^{13} \mathrm{~cm}^{-2}}$}} & \multirow{2}{*}{\multicolumn{2}{|c|}{$\frac{N(\mathrm{CO})}{10^{13} \mathrm{~cm}^{-2}}$}} & \multicolumn{2}{|c|}{$N(\mathrm{OH})$} \\
\hline & & & & & & & & & & & & & & $10^{13}$ & $\mathrm{~m}^{-2}$ \\
\hline & tot & S8 & S9 & $\mathrm{S} 8$ & S9 & obs & S8 & S9 & obs & $\mathrm{S} 8$ & S9 & S8 & S9 & S8 & S9 \\
\hline 55879 & 0.36 & 0.07 & 0.01 & 16 & 4 & 0.0 & 0.3 & 0.1 & 0.3 & 0.4 & 0.2 & 1.7 & 0.7 & 1.3 & 0.5 \\
\hline 53975 & 0.66 & 0.06 & 0.01 & 8 & 2 & 0.0 & 0.2 & 0.1 & 0.3 & 0.3 & 0.2 & 1.4 & 0.7 & 1.0 & 0.5 \\
\hline 53755 & 0.75 & 0.18 & 0.04 & 20 & 5 & 0.3 & 0.7 & 0.4 & 0.9 & 0.9 & 1.0 & 4.2 & 2.7 & 3.1 & 2.1 \\
\hline 54662 & 1.05 & 0.21 & 0.04 & 18 & 4 & 0.8 & 0.9 & 0.4 & 1.1 & 1.1 & 1.0 & 5.0 & 2.7 & 3.8 & 2.1 \\
\hline 52382 & 1.32 & 0.48 & 0.10 & 31 & 8 & 1.1 & 2.0 & 1.1 & 2.4 & 2.4 & 2.4 & 11.2 & 6.7 & 8.4 & 5.3 \\
\hline 63423 & 1.53 & 0.21 & 0.04 & 12 & 3 & 3.5 & 0.9 & 0.4 & 1.1 & 1.1 & 1.0 & 5.0 & 2.7 & 3.8 & 2.1 \\
\hline 62150 & 1.77 & 0.58 & 0.11 & 28 & 7 & 2.4 & 2.4 & 1.3 & 2.9 & 2.9 & 2.9 & 13.7 & 8.1 & 10.2 & 6.4 \\
\hline 61827 & 2.10 & 0.26 & 0.05 & 10 & 3 & 4.1 & 1.1 & 0.6 & 1.3 & 1.3 & 1.2 & 6.1 & 3.4 & 4.6 & 2.7 \\
\hline 62844 & 2.67 & 0.64 & 0.12 & 20 & 5 & 5.0 & 2.6 & 1.5 & 3.2 & 3.2 & 3.1 & 15.1 & 8.7 & 11.3 & 6.9 \\
\hline$-32^{\circ} 1734$ & 3.66 & 1.20 & 0.24 & 28 & 7 & 9.0 & 4.9 & 2.8 & 6.0 & 6.0 & 6.0 & 28.2 & 16.8 & 21.1 & 13.3 \\
\hline$-33^{\circ} 1768$ & 4.40 & 0.88 & 0.17 & 17 & 4 & 12.0 & 3.6 & 2.0 & 4.4 & 4.4 & 4.4 & 20.6 & 12.1 & 15.5 & 9.6 \\
\hline 63804 & 3.36 & 0.96 & 0.19 & 24 & 6 & 5.3 & 4.0 & 2.2 & 4.8 & 4.8 & 4.8 & 22.6 & 13.4 & 16.9 & 10.6 \\
\hline 78344 & 4.20 & 0.70 & 0.13 & 14 & 3 & 5.0 & 2.9 & 1.6 & 3.5 & 3.5 & 3.4 & 16.5 & 9.4 & 12.3 & 7.4 \\
\hline 74371 & 0.93 & 0.29 & 0.06 & 25 & 6 & 1.0 & 1.2 & 0.7 & 1.4 & 1.4 & 1.5 & 6.7 & 4.0 & 5.0 & 3.2 \\
\hline 75149 & 1.14 & 0.24 & 0.05 & 18 & 4 & 1.7 & 1.0 & 0.6 & 1.2 & 1.2 & 1.2 & 5.6 & 3.4 & 4.2 & 2.7 \\
\hline 74194 & 1.62 & 0.34 & 0.07 & 18 & 4 & 1.3 & 1.4 & 0.8 & 1.7 & 1.7 & 1.7 & 8.1 & 4.7 & 6.1 & 3.7 \\
\hline 75211 & 2.16 & 0.73 & 0.14 & 28 & 7 & 2.3 & 3.0 & 1.7 & 3.6 & 3.6 & 3.6 & 17.0 & 10.1 & 12.7 & 8.0 \\
\hline 76556 & 2.19 & 0.30 & 0.06 & 12 & 3 & 4.0 & 1.2 & 0.7 & 1.5 & 1.5 & 1.5 & 7.0 & 4.0 & 5.2 & 3.2 \\
\hline 75860 & 2.79 & 1.50 & 0.30 & 45 & 11 & 2.8 & 6.2 & 3.5 & 7.5 & 7.5 & 7.5 & 35.2 & 20.8 & 26.3 & 16.5 \\
\hline$-44^{\circ} 3129$ & 3.10 & 1.26 & 0.25 & 34 & 8 & 5.3 & 5.2 & 2.9 & 6.3 & 6.3 & 6.3 & 29.6 & 17.5 & 22.2 & 13.8 \\
\hline$-45^{\circ} 3218$ & 3.18 & 0.56 & 0.11 & 15 & 4 & 9.0 & 2.3 & 1.3 & 2.8 & 2.8 & 2.9 & 13.1 & 8.1 & 9.8 & 6.4 \\
\hline$-46^{\circ} 3272$ & 3.18 & 0.64 & 0.12 & 17 & 4 & 6.0 & 2.6 & 1.5 & 3.2 & 3.2 & 3.1 & 15.1 & 8.7 & 11.3 & 6.9 \\
\hline 73882 & 2.13 & 0.48 & 0.10 & 19 & 5 & 3.7 & 2.0 & 1.1 & 2.4 & 2.4 & 2.4 & 11.2 & 6.7 & 8.4 & 5.3 \\
\hline
\end{tabular}

Table 12. Modeled $\mathrm{CH}, \mathrm{CH}^{+}, \mathrm{CO}$, and $\mathrm{OH}$ column densities in the hot MHD component toward NGC 4755 and Cen OB1.

\begin{tabular}{|c|c|c|c|c|c|c|c|c|c|c|c|c|c|c|c|}
\hline \multirow{3}{*}{$\begin{array}{l}\text { star } \\
\mathrm{HD} / \mathrm{CPD}\end{array}$} & \multirow{2}{*}{\multicolumn{3}{|c|}{$A_{\mathrm{V}}(\mathrm{mag})$}} & \multirow{2}{*}{\multicolumn{2}{|c|}{$N_{\mathrm{s}}$}} & \multirow{2}{*}{\multicolumn{3}{|c|}{$N(\mathrm{CH})$}} & \multicolumn{3}{|c|}{$N\left(\mathrm{CH}^{+}\right)$} & \multicolumn{2}{|c|}{$N(\mathrm{CO})$} & \multicolumn{2}{|c|}{$N(\mathrm{OH})$} \\
\hline & & & & & & & & & & $3 \mathrm{~cm}$ & & $10^{13}$ & & $10^{13}$ & \\
\hline & tot & $\mathrm{S} 8$ & S9 & $\mathrm{S} 8$ & S9 & obs & $\mathrm{S} 8$ & $\mathrm{~S} 9$ & obs & $\mathrm{S} 8$ & S9 & $\mathrm{S} 8$ & S9 & $\mathrm{S} 8$ & S9 \\
\hline 111973 & 1.00 & 0.26 & 0.05 & 22 & 5 & 0.8 & 1.1 & 0.6 & 1.3 & 1.3 & 1.2 & 6.1 & 3.4 & 4.6 & 2.7 \\
\hline 111904 & 1.00 & 0.24 & 0.05 & 20 & 5 & 0.7 & 1.0 & 0.6 & 1.2 & 1.2 & 1.2 & 5.6 & 3.4 & 4.2 & 2.7 \\
\hline 111934 & 1.08 & 0.24 & 0.05 & 19 & 5 & 0.9 & 1.0 & 0.6 & 1.2 & 1.2 & 1.2 & 5.6 & 3.4 & 4.2 & 2.7 \\
\hline 111990 & 1.16 & 0.30 & 0.06 & 22 & 5 & 1.1 & 1.2 & 0.7 & 1.5 & 1.5 & 1.5 & 7.0 & 4.0 & 5.2 & 3.2 \\
\hline$-59^{\circ} 4551$ & 1.40 & 0.54 & 0.11 & 32 & 8 & 1.4 & 2.2 & 1.2 & 2.7 & 2.7 & 2.7 & 12.6 & 7.4 & 9.4 & 5.8 \\
\hline 114886 & 1.28 & 0.40 & 0.08 & 26 & 6 & 0.9 & 1.7 & 0.9 & 2.0 & 2.0 & 1.9 & 9.5 & 5.4 & 7.1 & 4.2 \\
\hline 115704 & 2.22 & 0.56 & 0.11 & 21 & 5 & 1.8 & 2.3 & 1.3 & 2.8 & 2.8 & 2.9 & 13.1 & 8.1 & 9.8 & 6.4 \\
\hline 112366 & 2.24 & 0.42 & 0.09 & 16 & 4 & 3.8 & 1.7 & 1.0 & 2.1 & 2.1 & 2.2 & 9.8 & 6.0 & 7.3 & 4.8 \\
\hline 115363 & 2.58 & 0.36 & 0.07 & 12 & 3 & 3.6 & 1.5 & 0.8 & 1.8 & 1.8 & 1.7 & 8.4 & 4.7 & 6.3 & 3.7 \\
\hline 110639 & 2.74 & 0.73 & 0.14 & 22 & 5 & 3.4 & 3.0 & 1.7 & 3.6 & 3.6 & 3.6 & 17.0 & 10.1 & 12.7 & 8.0 \\
\hline 114011 & 2.90 & 1.61 & 0.32 & 46 & 11 & 4.7 & 6.6 & 3.7 & 8.0 & 8.0 & 8.0 & 37.7 & 22.2 & 28.2 & 17.5 \\
\hline 113432 & 3.06 & 0.73 & 0.14 & 20 & 5 & 4.8 & 3.0 & 1.7 & 3.6 & 3.6 & 3.6 & 17.0 & 10.1 & 12.7 & 8.0 \\
\hline 113422 & 3.24 & 1.12 & 0.22 & 29 & 7 & 4.2 & 4.6 & 2.6 & 5.6 & 5.6 & 5.6 & 26.2 & 15.5 & 19.6 & 12.2 \\
\hline 112272 & 3.26 & 0.76 & 0.15 & 20 & 5 & 5.6 & 3.1 & 1.8 & 3.8 & 3.8 & 3.9 & 17.9 & 10.8 & 13.4 & 8.5 \\
\hline 114213 & 3.42 & 0.26 & 0.05 & 6 & 2 & 5.9 & 1.1 & 0.6 & 1.3 & 1.3 & 1.2 & 6.1 & 3.4 & 4.6 & 2.7 \\
\hline
\end{tabular}


Table 13. Predicted columns for $\mathrm{H}_{2}$ rotational levels in the shocked gas.

\begin{tabular}{|c|c|c|c|c|c|c|c|c|}
\hline \multirow[b]{2}{*}{ star } & \multirow[b]{2}{*}{$A_{\mathrm{V}}$} & \multicolumn{7}{|c|}{$\bar{N}\left(\mathrm{H}_{2}\right)\left(\mathrm{cm}^{-2}\right) V_{\mathrm{s}}=9 \mathrm{~km} \mathrm{~s}^{-1}$} \\
\hline & & $J=0$ & $J=1$ & $J=2$ & $J=3$ & $J=4$ & $J=5$ & $J=6$ \\
\hline HD 55879 & 0.36 & $1.3(18)$ & $4.0(18)$ & $2.2(17)$ & $8.5(16)$ & $2.4(15)$ & $2.2(14)$ & $1.5(12)$ \\
\hline HD 53975 & 0.66 & $1.3(18)$ & $4.0(18)$ & $2.2(17)$ & $8.5(16)$ & $2.4(15)$ & $2.2(14)$ & $1.5(12)$ \\
\hline HD 53755 & 0.75 & $5.2(18)$ & $1.6(19)$ & $8.9(17)$ & $3.4(17)$ & $9.7(15)$ & $8.9(14)$ & $6.0(12)$ \\
\hline HD 54662 & 1.05 & $5.2(18)$ & $1.6(19)$ & $8.9(17)$ & $3.4(17)$ & $9.7(15)$ & $8.9(14)$ & $6.0(12)$ \\
\hline HD 52382 & 1.32 & $1.3(19)$ & $4.0(19)$ & $2.2(18)$ & $8.5(17)$ & $2.4(16)$ & $2.2(15)$ & $1.5(13)$ \\
\hline HD 63423 & 1.53 & $5.2(18)$ & $1.6(19)$ & $8.9(17)$ & $3.4(17)$ & $9.7(15)$ & $8.9(14)$ & $6.0(12)$ \\
\hline HD 62150 & 1.77 & $1.5(19)$ & $4.8(19)$ & $2.7(18)$ & $1.0(18)$ & $2.9(16)$ & $2.7(15)$ & $1.8(13)$ \\
\hline HD 61827 & 2.10 & $6.5(18)$ & $2.0(19)$ & $1.1(18)$ & $4.2(17)$ & $1.2(16)$ & $1.1(15)$ & $7.4(12)$ \\
\hline HD 62844 & 2.67 & $1.7(19)$ & $5.2(19)$ & $2.9(18)$ & 1.1(18) & $3.2(16)$ & $2.9(15)$ & $1.9(13)$ \\
\hline $\mathrm{CPD}-32^{\circ} 1734$ & 3.66 & $3.2(19)$ & $1.0(20)$ & $5.5(18)$ & $2.1(18)$ & $6.1(16)$ & $5.6(15)$ & $3.7(13)$ \\
\hline $\mathrm{CPD}-33^{\circ} 1768$ & 4.40 & $2.3(19)$ & $7.2(19)$ & $4.0(18)$ & $1.5(18)$ & $4.4(16)$ & $4.0(15)$ & $2.7(13)$ \\
\hline HD 63804 & 3.36 & $2.6(19)$ & $8.0(19)$ & $4.4(18)$ & $1.7(18)$ & $4.9(16)$ & $4.5(15)$ & $3.0(13)$ \\
\hline HD 78344 & 4.20 & $1.8(19)$ & $5.6(19)$ & $3.1(18)$ & $1.2(18)$ & $3.4(16)$ & $3.1(15)$ & $2.1(13)$ \\
\hline HD 74371 & 0.93 & $7.7(18)$ & $2.4(19)$ & $1.3(18)$ & $5.1(17)$ & $1.5(16)$ & $1.3(15)$ & $8.9(12)$ \\
\hline HD 75149 & 1.14 & $6.5(18)$ & $2.0(19)$ & $1.1(18)$ & $4.2(17)$ & $1.2(16)$ & $1.1(15)$ & $7.4(12)$ \\
\hline HD 74194 & 1.62 & $9.0(18)$ & $2.8(19)$ & $1.6(18)$ & $5.9(17)$ & $1.7(16)$ & $1.6(15)$ & $1.0(13)$ \\
\hline HD 75211 & 2.16 & $1.9(19)$ & $6.0(19)$ & $3.3(18)$ & $1.3(18)$ & $3.6(16)$ & $3.3(15)$ & $2.2(13)$ \\
\hline HD 76556 & 2.19 & $7.7(18)$ & $2.4(19)$ & $1.3(18)$ & $5.1(17)$ & $1.5(16)$ & $1.3(15)$ & $8.9(12)$ \\
\hline HD 75860 & 2.79 & $4.0(19)$ & $1.2(20)$ & $6.9(18)$ & $2.6(18)$ & $7.5(16)$ & $6.9(15)$ & $4.6(13)$ \\
\hline $\mathrm{CPD}-44^{\circ} 3129$ & 3.10 & $3.4(19)$ & $1.0(20)$ & $5.8(18)$ & $2.2(18)$ & $6.3(16)$ & $5.8(15)$ & $3.9(13)$ \\
\hline $\mathrm{CPD}-45^{\circ} 3218$ & 3.18 & $1.5(19)$ & $4.8(19)$ & $2.7(18)$ & $1.0(18)$ & $2.9(16)$ & $2.7(15)$ & $1.8(13)$ \\
\hline $\mathrm{CPD}-46^{\circ} 3272$ & 3.18 & $1.7(19)$ & $5.2(19)$ & $2.9(18)$ & $1.1(18)$ & $3.2(16)$ & $2.9(15)$ & $1.9(13)$ \\
\hline HD 73882 & 2.13 & $1.3(19)$ & $4.0(19)$ & $2.2(18)$ & $8.5(17)$ & $2.4(16)$ & $2.2(15)$ & $1.5(13)$ \\
\hline HD 111973 & 1.00 & $6.5(18)$ & $2.0(19)$ & $1.1(18)$ & $4.2(17)$ & $1.2(16)$ & $1.1(15)$ & $7.4(12)$ \\
\hline HD 111904 & 1.00 & $6.5(18)$ & $2.0(19)$ & $1.1(18)$ & $4.2(17)$ & $1.2(16)$ & $1.1(15)$ & $7.4(12)$ \\
\hline HD 111934 & 1.08 & $6.5(18)$ & $2.0(19)$ & $1.1(18)$ & $4.2(17)$ & $1.2(16)$ & $1.1(15)$ & $7.4(12)$ \\
\hline HD 111990 & 1.16 & $7.7(18)$ & $2.4(19)$ & $1.3(18)$ & $5.1(17)$ & $1.5(16)$ & $1.3(15)$ & $8.9(12)$ \\
\hline $\mathrm{CPD}-59^{\circ} 4551$ & 1.40 & $1.4(19)$ & $4.4(19)$ & $2.4(18)$ & $9.3(17)$ & $2.7(16)$ & $2.5(15)$ & $1.6(13)$ \\
\hline HD 114886 & 1.28 & $1.0(19)$ & $3.2(19)$ & $1.8(18)$ & $6.8(17)$ & $1.9(16)$ & $1.8(15)$ & $1.2(13)$ \\
\hline HD 115704 & 2.22 & $1.5(19)$ & $4.8(19)$ & $2.7(18)$ & $1.0(18)$ & $2.9(16)$ & $2.7(15)$ & $1.8(13)$ \\
\hline HD 112366 & 2.24 & $1.2(19)$ & $3.6(19)$ & $2.0(18)$ & $7.6(17)$ & $2.2(16)$ & $2.0(15)$ & $1.3(13)$ \\
\hline HD 115363 & 2.58 & $9.0(18)$ & $2.8(19)$ & $1.6(18)$ & $5.9(17)$ & $1.7(16)$ & $1.6(15)$ & $1.0(13)$ \\
\hline HD 110639 & 2.74 & $1.9(19)$ & $6.0(19)$ & $3.3(18)$ & $1.3(18)$ & $3.6(16)$ & $3.3(15)$ & $2.2(13)$ \\
\hline HD 114011 & 2.90 & $4.3(19)$ & $1.3(20)$ & $7.3(18)$ & $2.8(18)$ & $8.0(16)$ & $7.4(15)$ & $4.9(13)$ \\
\hline HD 113432 & 3.06 & $1.9(19)$ & $6.0(19)$ & $3.3(18)$ & $1.3(18)$ & $3.6(16)$ & $3.3(15)$ & $2.2(13)$ \\
\hline HD 113422 & 3.24 & $3.0(19)$ & $9.2(19)$ & $5.1(18)$ & $1.9(18)$ & $5.6(16)$ & $5.1(15)$ & $3.4(13)$ \\
\hline HD 112272 & 3.26 & 2.1(19) & $6.4(19)$ & $3.6(18)$ & $1.4(18)$ & $3.9(16)$ & $3.6(15)$ & $2.4(13)$ \\
\hline HD 114213 & 3.42 & $6.5(18)$ & $2.0(19)$ & $1.1(18)$ & $4.2(17)$ & $1.2(16)$ & $1.1(15)$ & $7.4(12)$ \\
\hline
\end{tabular}

the gas toward HD 62150, HD 75149, and CPD-443129. For more reddened sight lines such as toward HD 63804, HD 78344, and HD 75211, $N\left(\mathrm{C}_{2}\right)$ and $N(\mathrm{CN})$ are nearly independent of density because production and destruction involve collisions.

We also determined column densities in the $J^{\prime \prime}=0-6$ rotation levels in $v^{\prime \prime}=0$ of the electronic ground state of molecular hydrogen. Predicted column densities are given in Table 13 in Cols. 3-9. The syntax $\mathrm{x}(\mathrm{nn})$ used in that table describes values of $\mathrm{x} \times 10^{\mathrm{nn}}$. These predictions of $\mathrm{H}_{2}$ columns from our models can be tested through future FUSE observations. One star, HD 73882, has been observed with FUSE (Snow et al. 2000). The total hot component of $\mathrm{H}_{2}\left(J^{\prime \prime}>1\right)$ found in our model is similar to the observations, although the population density in the highest levels $\left(J^{\prime \prime}>4\right)$ is larger than our predictions.
The $\mathrm{H}_{2}$ excitation temperature toward HD 73882 from the MHD model is $200 \mathrm{~K}$, compared to a value of $300 \mathrm{~K}$ inferred from the observations. Either the MHD models do not heat the gas sufficiently, or the high- $J$ values are populated primarily by UV pumping from the O8.5 star as claimed by Snow et al. (2000).

\section{Summary}

We present $\mathrm{CN}$ observations for the three southern translucent clouds which obscure stars in the NGC 2439, Vela OB1, and Cen OB1 associations. The CN data complement previous detections of $\mathrm{CH}, \mathrm{CH}^{+}$, and $\mathrm{C}_{2}$. Altogether, we present a homogeneous set of $\mathrm{CH}, \mathrm{CH}^{+}$, $\mathrm{CN}$, and $\mathrm{C}_{2}$ column densities and radial velocities. We use 
the CN data to study its production via chemical models. Our main results are:

- The observed column densities of $\mathrm{CN}$ and $\mathrm{C}_{2}$ are well reproduced by our chemical reaction network of quiescent molecular gas.

- Most lines of sight through the three translucent clouds test diffuse material, characterised by densities of a few hundred $\mathrm{cm}^{-3}$ and ultraviolet radiation fields 15 times the average interstellar flux. The densities inferred here from $\mathrm{CN}$ are in excellent agreement with previously inferred densities from $\mathrm{C}_{2}$ excitation.

- The radial velocities of $\mathrm{CN}$ agree with those of $\mathrm{CH}$, $\mathrm{CH}^{+}, \mathrm{C}_{2}$, and $\mathrm{CO}$.

- An additional gas component containing 10-50 MHD shocks in each line of sight explains the observed column densities of $\mathrm{CH}^{+}$and 10 to $30 \%$ of the observed $\mathrm{CH}$ column.

Acknowledgements. SRF was supported in part by NASA LTSA grant NAG5-4957. It is a pleasure to thank the referee, Dr. Steve Fossey, for his thorough review of the paper and his valuable comments to improve the manuscript.

\section{References}

Bauschlicher, C. W. Jr., Langhoff, S. R., \& Taylor, P. R. 1988, ApJ, 332, 531

Black, J. H., \& van Dishoeck, E. F. 1988, ApJ, 331, 986

Black, J. H., \& van Dishoeck, E. F. 1991, ApJL, 369, L9

Le Bourlot, J., Pineau des Forêts, G., Roueff, E., \& Schilke, P. 1993, ApJ, 416, L87

Bucher, M. E., \& Glinski, R. J. 1999, MNRAS, 308, 29

Crane, P., Lambert, D. L., \& Sheffer, Y. 1995, ApJS, 99, 107

Crawford, I. 1995, MNRAS, 277, 458

Crawford, I. 1997, MNRAS, 290, 41

Davis, S. P., Shortenhaus, D., Stark, G., et al. 1986, ApJ, 303, 892

Duley, W. W., Hartquist, T. W., Sternberg, A., Wagenblast, R., \& Williams, D. A. 1992, MNRAS, 255, 463

Draine, B. T., \& Katz, N. 1986, ApJ, 310, 392

Falgarone, E., Pineau des Forets, G., \& Roueff, E. 1995, A\&A, 300,870

Federman, S. R., Danks, A. C., \& Lambert, D. L. 1984, ApJ, 287,219
Federman, S. R., \& Lambert, D. L. 1988, ApJ, 328, 777

Federman, S. R., Knauth, D. C., Lambert, D. L., \& Andersson, B.-G. 1997a, ApJ, 489, 758

Federman, S. R., Strom, C. J., Lambert, D. L., et al. 1994, ApJ, 424, 772

Federman, S. R., Welty, D. E., \& Cardelli, J. A. 1997b, ApJ, 481, 795

Fixsen, D. J., Mather, J. C., Shafer, R. A., Brodd, S., \& Jensen, K. A. 1997, AAS, 191, 91.05

Flower, D. R., \& Pineau des Forêts, G. 1998, MNRAS, 297, 1182

Gredel, R. 1997, A\&A, 320, 929, Paper I

Gredel, R. 1999, A\&A, 351, 657

Gredel, R., van Dishoeck, E. F., \& Black, J. F. 1991, A\&A, 251,625

Gredel, R., van Dishoeck, E. F., \& Black, J. F. 1993, A\&A, 269,477

Jenkins, E. B. 1986, ApJ, 304, 739

Joseph, C. L., Snow, T. P., Jr., Seab, C. G., \& Crutcher, R. M. 1986, ApJ, 309, 771

Joulain, K., Falgarone, E., Pineau des Forêts, G., \& Flower, D. 1998, A\&A, 340, 241

Knauth, D. C., Federman, S. R., Pan, K., Yan, M., \& Lambert, D. L. 2001, ApJS, 135, 201

Lambert, D. L., Sheffer, Y., \& Crane, P. 1990, ApJ, 359, L19

Meyer, D. M. 1997, in Molecules in Astrophysics: Probes and Processes, ed. E. F. van Dishoeck (Kluwer), 407

Meyer, D. M., Cardelli, J. A., \& Sofia, U. J. 1997, ApJ, 490, L103

Meyer, D. M., Jura, M., \& Cardelli, J. A. 1998, ApJ, 493, 222

Münch, G. 1968, in Nebulae \& Interstellar Matter, ed. B. M. Middlehurst, \& L. H. Aller (Univ. Chicago Press), 365

Pan, K., Federman, S. R., \& Welty, D. E. 2001, ApJ, 558, L105

Pineau des Forêts, G., Flower, D. R., Hartquist, T. W., \& Dalgarno, A. 1986, MNRAS, 220, 801

Prasad, C. V. V., Bernath, P. F., Frum, C., \& Engleman, R. Jr. 1992, J. Molec. Spec., 151, 459

Reed Cameron, B. 2000, AJ, 119, 1855

Roth, K. C., \& Meyer, D. M. 1995, ApJ, 441, 129

Snow, T. P., et al. 2000, ApJ, 538, L65

Sofia, U. J., Cardelli, J. A., Guerin, K. P., \& Meyer, D. M. 1997, ApJ, 482, L105

Spaans, M. 1995, Ph.D. Thesis, Models of Inhomogeneous Interstellar Clouds (Leiden)

Strömgren, B. 1948, ApJ, 108, 242

van Dishoeck, E. F., \& Black, J. H. 1989, ApJ, 340, 273

Zsargó, J. 2000, Ph.D. Dissertation, Univ. of Toledo 